\title{
Superconducting Detectors and Mixers for Millimeter and Submillimeter Astrophysics
}

\author{
JONAS ZMUIDZINAS, MEMBER, IEEE, AND PAUL L. RICHARDS
}

\section{Invited Paper}

\begin{abstract}
Superconducting detectors will play an increasingly significant role in astrophysics, especially at millimeter through far-IR wavelengths, where the scientific opportunities include key problems in astronomy and cosmology. Superconducting detectors offer many benefits: outstanding sensitivity, lithographic fabrication, and large array sizes, especially through the recent development of multiplexing techniques. This paper describes the scientific opportunities, the basic physics of these devices, the techniques for radiation coupling, and reviews the recent progress in direct detectors, such as transition-edge bolometers, and the work on tunnel junction (superconductor-insulator-superconductor) and hot-electron mixers.
\end{abstract}

Keywords-Astronomy, millimeter-wave detectors, radio astronomy, submillimeter-wave detectors, superconducting devices, superconducting radiation detectors, superconductor-insulator-superconductor mixers.

\section{INTRODUCTION}

Superconducting detectors are finding a key role in astrophysics at far-IR to millimeter wavelengths, where the early universe radiates very strongly. For this paper, we will loosely define the millimeter band as $10 \mathrm{~mm}>\lambda>1 \mathrm{~mm}$, the submillimeter band as $1 \mathrm{~mm}>\lambda>0.2 \mathrm{~mm}$, and the far-IR as $200 \mu \mathrm{m}>\lambda>20 \mu \mathrm{m} .{ }^{1}$ These correspond to fre-

Manuscript received December 12, 2003; revised April 19, 2004. This work was supported in part by A. Lidow, a California Institute of Technology (Caltech), Pasadena, Trustee; in part by the National Aeronautics and Space Administration (NASA), through Aerospace Technology and Space Science Enterprises; and in part by the National Science Foundation, and the Stratospheric Observatory for Infrared Astronomy (SOFIA) program, administered by the Universities Space Research Organization (USRA) for NASA.

J. Zmuidzinas is with the Division of Physics, Mathematics, and Astronomy, California Institute of Technology, Pasadena, CA 91125 USA (e-mail: jonas@submm.caltech.edu).

P. L. Richards is with the University of California, Berkeley, CA 94720 USA.

Digital Object Identifier 10.1109/JPROC.2004.833670

${ }^{1}$ The choice of $\lambda=200 \mu \mathrm{m}$ as the boundary between the submillimeter and far-IR coincides with the long-wavelength cutoff of stressed Ge: Ga photoconductor detectors, currently the most commonly used detector technology for far-IR astronomy. quency ranges of $30 \mathrm{GHz}<\nu<300 \mathrm{GHz}, 300 \mathrm{GHz}<\nu<$ $1500 \mathrm{GHz}$, and $1.5 \mathrm{THz}<\nu<15 \mathrm{THz}$, respectively.

Two generic approaches are used for astrophysical measurements. Measurements with low spectral resolution $\lambda / \Delta \lambda$ of 3-10 are called photometry. They are used to characterize broad spectrum sources such as electron synchrotron and thermal bremstrahlung emission, and thermal emission from interstellar dust grains. Spectroscopy refers to measurements with higher resolution, up to $10^{6}$, that are typically used to characterize molecular and atomic spectral lines.

\section{A. Photometry}

Photometry at millimeter and submillimeter wavelengths plays a key role in astrophysics. For example, photometry is used to study the cosmic microwave background (CMB), which lights up the sky at millimeter wavelengths and makes up most of the electromagnetic energy in the universe. $\mathrm{CMB}$ measurements provide us a snapshot of the early universe 300000 years after the Big Bang. Measurements of the anisotropy of this radiation have already shown that the geometry of the universe is Euclidean (space is flat) [1]-[3], have provided the most accurate values for several important cosmological parameters [4], and have provided very strong support for an inflation-motivated cosmological model dominated by dark energy and containing substantial dark matter. Future measurements of the polarization of the $\mathrm{CMB}$ are expected to yield detailed information about the universe at the time of inflation (around $10^{-38} \mathrm{~s}$ !) and particle physics on the energy scale of Grand Unification [5]-[8]. Also, surveys of the scattering of the CMB as it passes through clusters of galaxies (Sunyaev-Zeldovich effect) promise to locate thousands of clusters of galaxies at all distances where they exist [9]. These data will test the history of the expansion of the universe, the formation of structure, and even gravity on cosmological length scales. A future $\mathrm{CMB}$ polarization space mission has been proposed that will use superconducting detectors. 
Another example is the study of interstellar dust emission from galaxies. The best known signal from galaxies is the thermal emission from stars at visible and UV wavelengths, which is measured using charge-coupled device (CCD) arrays. It has recently been discovered that for some of the earliest, very distant galaxies, most of this starlight does not reach us [10]-[14]. The starlight is instead absorbed by dust and reradiated at longer wavelengths. In fact, the total amount of energy in submillimeter light in the universe is about equal to that in the UV/visible/near-IR band [15]-[17]. Furthermore, the submillimeter band is unique in that galaxies may be seen out to very large distances, because the dust emission spectrum has a very steep long-wavelength slope $\left(\sim \nu^{3.5}\right)$, so that the cosmological frequency redshift essentially compensates for the dimming due to the increasing distance. Measurements of this dust emission by submillimeter photometry give us the total luminosity of the galaxy, which is related to the rate at which stars are forming, and also provides information about the behavior of the dust. A future 10-m class space telescope has been proposed to exploit this scientific opportunity [18].

The detector technologies used for photometry depend on the wavelength. Cooled high electron mobility transistor (HEMT) amplifiers, followed by diode detectors, are often used for $\lambda>3 \mathrm{~mm}$ [19]. Thermal direct detectors, especially semiconducting bolometers [20]-[22] are in current use for $3>\lambda>0.2 \mathrm{~mm}$. Semiconducting photon detectors are generally used for wavelengths $\lambda<0.2 \mathrm{~mm}$ [23].

A new generation of bolometer is being developed which uses a superconducting transition-edge sensor (TES) element as the thermistor [24]. This approach lends itself to focal planes of thousands of pixels and is replacing semiconducting bolometers in systems now under construction. Also, prototypes exist for superconducting photon detectors operating in the same wavelength range. These generally have less stringent requirements for cooling than bolometers and, thus, may become the technology of choice at some future time.

\section{B. Spectroscopy}

The measurement of spectral lines also plays a critical role in astrophysics at millimeter and submillimeter wavelengths. Molecular rotation lines dominate at millimeter and submillimeter wavelengths and atomic fine structure lines at far-IR wavelengths [25]. Resolutions as large as $\lambda / \Delta \lambda \sim 10^{6}$ are often needed to measure Doppler shifts and spectral line profiles. Direct detection instruments such as bolometers behind grating or Fabry-Pérot spectrometers [26]-[30] are used at moderate resolution, while heterodyne receiver systems using superconducting mixers as frequency downconverters are used for higher spectral resolution $\left(\lambda / \Delta \lambda>10^{3}\right)$ [31]-[35]. These include the superconductor-insulator-superconductor (SIS) tunnel junction mixer for $3>\lambda>0.25 \mathrm{~mm}$ and hot-electron bolometer (HEB) mixers at shorter wavelengths.

Heterodyne receivers are quite familiar to most electrical engineers: an RF signal picked up by an antenna at frequency $\nu_{\mathrm{RF}}$ and the sine-wave output of a "local" oscillator (LO) at frequency $\nu_{\mathrm{LO}}$ are combined in a nonlinear device known as a mixer, which generates the beat frequency $\nu_{\mathrm{IF}}=\mid \nu_{\mathrm{RF}}-$ $\nu_{\mathrm{LO}} \mid$, also known as the intermediate frequency (IF). The IF signal may then be further downconverted or demodulated. For radioastronomical spectroscopy, the IF signal is processed by a multichannel spectrum analyzer, such as a filter bank, acoustooptical spectrometer (AOS) [36], or a digital correlator [37]-[41]. The IF spectrum is a replica of the original RF spectrum, and very high spectral resolution is possible, since $\nu_{\mathrm{IF}} \ll \nu_{\mathrm{RF}}$.

Typically, radio or microwave mixers are implemented using semiconductor diodes [42], e.g., GaAs Schottky diodes, and are not particularly low noise. Thus, the antenna signal is usually sent through a low-noise amplifier (LNA) prior to mixing, so that the noise and conversion efficiency of the mixer is not critical for the overall receiver system performance. Indeed, cryogenic HEMT amplifiers are widely used for radio astronomy at centimeter wavelengths [19]. However, at very high frequencies, in the millimeter through far-IR bands, LNAs do not exist, and so very sensitive first-stage mixers are needed. In addition, the output power of tunable submillimeter LO sources is generally quite low [33], so mixers with low LO power requirements are needed. Cryogenically cooled semiconductor diodes have been used for first-stage mixers in the past [43]-[46], although by now they have been replaced by superconducting tunnel junction (SIS) or HEB mixers.

The use of direct detection instead of heterodyne detection is highly desirable for moderate spectral resolution when the photon background is low [26], [47]. This is because heterodyne systems have a fundamental minimum noise floor known as the quantum limit [48], [49] which does not apply to direct detection systems. However, there are a number of technical challenges which make it preferable to use heterodyne receiver systems when high spectral resolution $(R=$ $\lambda / \Delta \lambda \sim 10^{5}-10^{6}$ ) is needed [50]-[52] at long wavelengths.

Another key application for superconducting heterodyne receivers is aperture synthesis interferometry using a multiple telescope array, which allows much higher spatial resolution images to be obtained than when using a single telescope [53]-[55]. In this technique, the downconverted signals from the telescopes are brought together, typically using optical fiber transmission, and are cross correlated [40], [41]. ALMA, which at present is the world's largest radio astronomy project, will be a 64-element array of $12-\mathrm{m}$ telecopes located on a high plateau in the Atacama desert in Chile [56], operating at frequencies between 30 and $950 \mathrm{GHz}$. The development of superconducting SIS receivers made this project possible; in fact, large arrays were already being contemplated in the early 1980s [57]-[59], only a few years after the very first SIS receivers were demonstrated (in 1979).

Submillimeter spectroscopy and interferometry are very powerful tools for the study of star and planet formation. Stars are formed by gravitational collapse deep inside cold molecular gas clouds, which also contain large amounts of dust which makes them opaque at visible or near-IR wavelengths. Most of the gas in molecular clouds is cool, 
$\sim 10-20 \mathrm{~K}$, and emits primarily at millimeter wavelengths. However, the radiation from newly formed stars heats the surrounding gas and dust, evaporating ices from the dust grains, which drives a complex network of chemical reactions and results in a vast forest of bright submillimeter spectral line emission [60]-[62]. Water is of course a particularly interesting species to study. Understandably, the earth's atmosphere is opaque at the rotational transition frequencies of water [63], so observations of interstellar water generally require airborne [32], [64] or space [31], [65], [66] observatories.

Newly born stars are thought to be formed from and surrounded by disks containing gas and dust, which may also later form planets. These disks are illuminated by ultraviolet light from the star, which causes the gas in the disk to heat up and emit strong lines in the submillimeter [67]. Millimeter and submillimeter interferometry are especially useful for studying the physical structure and dynamics of these disks [68]. It is even possible that at the end of its life, a dying star undergoes an episode in which a large increase in its luminosity evaporates the water ice from the comet-like debris left over from the disk, a process which is observable in the submillimeter range [69].

Star formation processes may also be studied at larger scales using long-wavelength observations of galaxies. Particularly interesting are the "starburst" or "ultraluminous IR" galaxies (ULIRGs) [70], in which a galaxy collision or merger compresses and shocks the interstellar gas and triggers an episode of rapid star formation. Submillimeter and far-IR spectroscopy [71]-[76] provides valuable information about the warm molecular and atomic gas which is heated by the newly formed massive stars (or the supernova explosions which follow!), while interferometry [77] provides detailed images of the central regions of these galaxies, where much of the activity takes place. Similar processes may explain the properties of the submillimeter-bright galaxies at high redshifts [78]-[80]. In the future, it may be possible to study the physics of these distant galaxies using very sensitive, moderate resolution direct-detection spectroscopy [26] with a cold space telescope [18].

\section{TRANSITION-EDGE BOLOMETERS}

\section{A. Introduction}

A TES bolometer for millimeter and submillimeter wavelength astrophysics consists of a radiation absorbing element attached to a thin superconducting film with a transition temperature $T_{c}$, which is weakly coupled to a heat sink at temperature $T_{0} \sim T_{c} / 2$. This TES is heated by a constant voltage bias to an operating point within the superconducting transition. The current through the TES is coupled to a superconducting quantum interference device (SQUID) which acts as a low noise current amplifier. In one typical device architecture, called a leg-isolated bolometer, the absorber and TES are deposited on a very thin membrane of Si or low-stress silicon nitride (LSN) for low heat capacity. The membrane is patterned into narrow legs which provide mechanical support with negligible thermal conductance. Metallic leads deposited on the legs provide electrical connection to the TES and the thermal conductance between the TES and the heat sink.

\section{B. Device Physics}

Superconducting bolometers have been known for 60 years, but rarely used. Traditionally, the sensor was biased with a constant current and read out with a voltage amplifier. The bias power $P_{B}=I^{2} R$ then increased with temperature due to the increase of resistance $R$ near $T_{c}$. This produced a positive electrothermal feedback which led to instability and even thermal runaway. The new idea due to Irwin [81] is to use a constant voltage bias to give a negative electrothermal feedback which stabilizes the temperature of the TES at the operating point on the transition. This voltage-biased TES bolometer has many of the traditional advantages of a transducer with large negative feedback including linearity, bandwidth, and immunity of the response to changes in external parameters such as the absorbed optical power and the temperature of the heat sink. These devices are produced entirely by thin-film deposition and optical lithography. Consequently, they are suitable for large format arrays.

In practice, the thermal conductance from the TES to the heat sink is chosen so that with no bias, the largest anticipated optical power $P_{\max }$ will heat the TES to $T_{c}$. The bias voltage $V$ is chosen so that for small optical power $P$, the TES will heat to a steep point on the transition. For intermediate values of $P$, the electrothermal feedback keeps the total power input $P+V^{2} / R$ (and, thus, the temperature) constant. The response of the bolometer current $I$ to a change in the optical power is called the current responsivity $S_{I}$. For a thermal circuit with a single pole response [82]

$$
S_{I} \equiv \frac{\partial I}{\partial P}=-\frac{1}{V_{B}} \frac{\mathcal{L}}{\mathcal{L}+1} \frac{1}{1+i \omega \tau}
$$

where $\mathcal{L}=\alpha P / G T$ is the loop gain, $\alpha=(T / R) d R / d T$ is a measure of the steepness of the superconducting transition, $G=d P / d T$ is the differential thermal conductance and $\tau$ is the effective time constant. For a typical loop gain $\mathcal{L} \sim 10^{3}$, the low-frequency responsivity becomes $S_{I}=-1 / V_{B}$, and depends only on the bias voltage and is independent of the signal power and the heat sink temperature. The effective time constant $\tau=\tau_{0} / \mathcal{L}$ is much shorter than the time constant without feedback $\tau_{0}=C / G$. In practice, the full reduction in $\tau$ is rarely seen because most devices do not have a single pole thermal circuit. For example, delay in the transfer of heat from the absorber to the TES (an internal time constant) is not reduced by feedback and typically produces time constants of a few milliseconds.

As shown in Fig. 1, the TES is transformer coupled to the SQUID by an input coil. A current-biased shunt resistor is used to provide a constant voltage bias to the TES. When the shunt resistor is operated close to the detector temperature, this configuration gives negligible Johnson noise from 


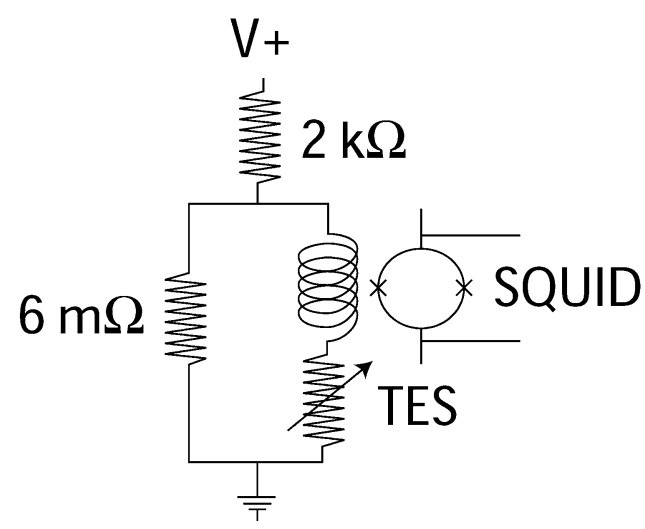

Fig. 1. Typical TES bias circuit. The voltage bias is achieved by current bias of a cold shunt resistor whose $\sim 10 \mathrm{~m} \Omega$ resistance is much smaller than the $R \sim 1 \Omega$ resistance of the TES. The current through the TES is measured with a SQUID ammeter, and the in-band reactance of the SQUID input inductor is much less than $R$.

the bias network. The SQUID readout has a number of advantages over the JFET amplifier used with a conventional semiconducting bolometer. It can operate at or near the bolometer temperature, has very low power dissipation, and has large noise margin. Also, the low characteristic impedances make the TES/SQUID system much less sensitive to microphonic pickup than conventional high-impedance bolometers with JFET readouts. The films and lithography used to produce SQUIDS are similar to those for TES bolometers, so integration on the same chip can be considered.

The current noise of the bolometer with no optical input can be written as a quadrature sum of contributions from thermal fluctuation noise, Johnson noise, and SQUID noise referred to the SQUID input [82]. At frequencies below $\omega=$ $1 / \tau$, thermal fluctuation noise usually dominates. At higher frequencies, Johnson noise dominates. Both of these noise sources decrease with decreasing temperature. The SQUID noise is typically negligible when it is properly coupled.

The most sensitive measurements of an astrophysical signal are made by minimizing the optical power from warm parts of the apparatus and by making the noise sources from the bolometer small compared with the photon noise from the total optical power $P$. For ground-based telescopes, $P$ contains contributions from the atmosphere and the ambient temperature telescope optics in addition to the astronomical signal. Photon noise limited operation can generally be achieved in photometric bandwidths by bolometers with a noise equivalent power (NEP) $\sim 10^{-17} \mathrm{~W} \cdot \mathrm{Hz}^{-1 / 2}$ which can be obtained by cooling to $T_{0}=0.25 \mathrm{~K}$ with a ${ }^{3} \mathrm{He}$ sorption refrigerator. For space experiments with cooled optics, an NEP $\sim 10^{-18} \mathrm{~W} \cdot \mathrm{Hz}^{-1 / 2}$ is needed for photometry, which can be obtained by cooling to $\sim 0.1 \mathrm{~K}$ with dilution or magnetic refrigerators. As discussed in Section I-B, moderate resolution direct-detection spectroscopy with a cold space telescope is of considerable scientific interest, but will require NEP $\sim 10^{-20} \mathrm{~W} \cdot \mathrm{Hz}^{-1 / 2}$, which is difficult to achieve with present technology.

Bolometric receivers require filters to define the required photometric bands. Since many astronomical sources are brighter at shorter wavelengths, it is particularly difficult to

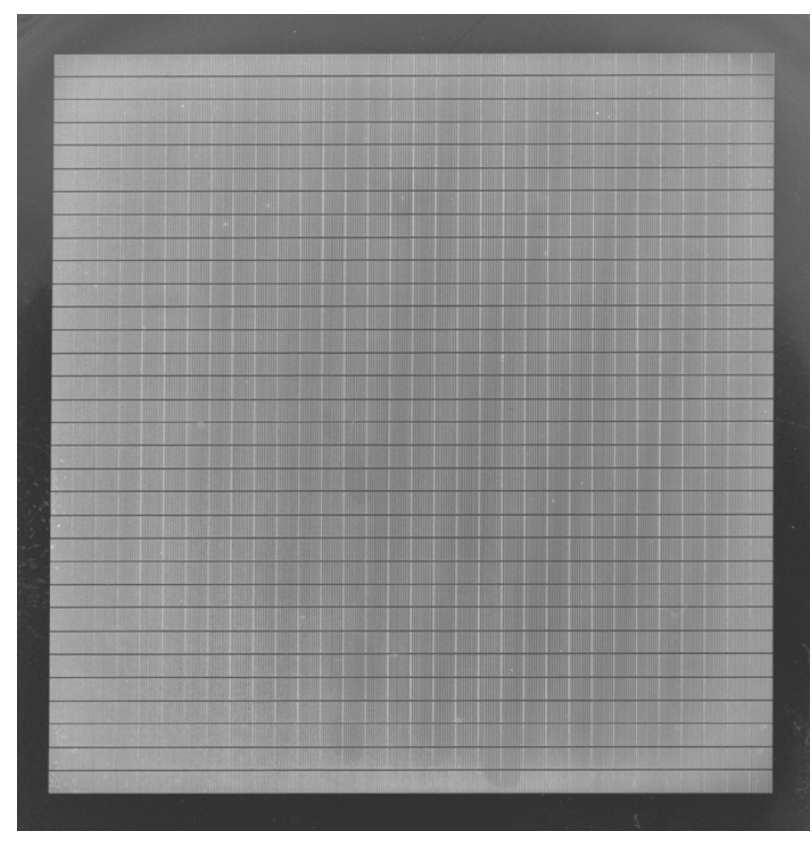

Fig. 2. Prototype for a 1024 pixel close-packed bolometer array. Each small square contains a freestanding LSN mesh supported on legs. Sensors and metallization have not yet been added.

block unwanted shorter wavelength radiation. Combinations of cold multilayer metal mesh filters and cold absorption filters made from various materials are used. Not all of the in-band optical power incident on a bolometric receiver is absorbed in the bolometers; optical efficiencies typically range from $40 \%$ to $80 \%$.

\section{TES Calorimeters}

The voltage-biased TES concept finds applications outside of the millimeter-wave bolometers described in this paper. Related devices have been developed as photon detectors from optical to gamma ray wavelengths [83]-[86]. The characteristic time for a photon to deposit its energy in the absorber is short compared to the thermal relaxation time of the device, so the height of the pulse is proportional to the energy of the absorbed photon. Detectors operated in this mode are called calorimeters. In addition to measuring the photon flux, they can obtain the spectrum of the source with high resolution. Individual TES calorimeters are used for laboratory X-ray spectroscopy [87], [88]. Large arrays of TES X-ray calorimeters are being developed for spectroscopic imaging on major astrophysical space missions [89], [90].

\section{TES Bolometer Designs}

The range of wavelengths where TES bolometers will be used lies between the millimeter wave technology of antennas and monolithic microwave integrated circuit (MMIC) amplifiers and the IR technology of close-packed arrays of photon detectors. The fabrication technologies used for TES bolometers are very flexible and specialized detectors are being developed to meet the needs of specific observations.

The 1024-pixel bolometer array structure shown in Fig. 2 is a step in the development of close-packed arrays to be placed in the focal plane of a far-IR telescope [91]. A single 


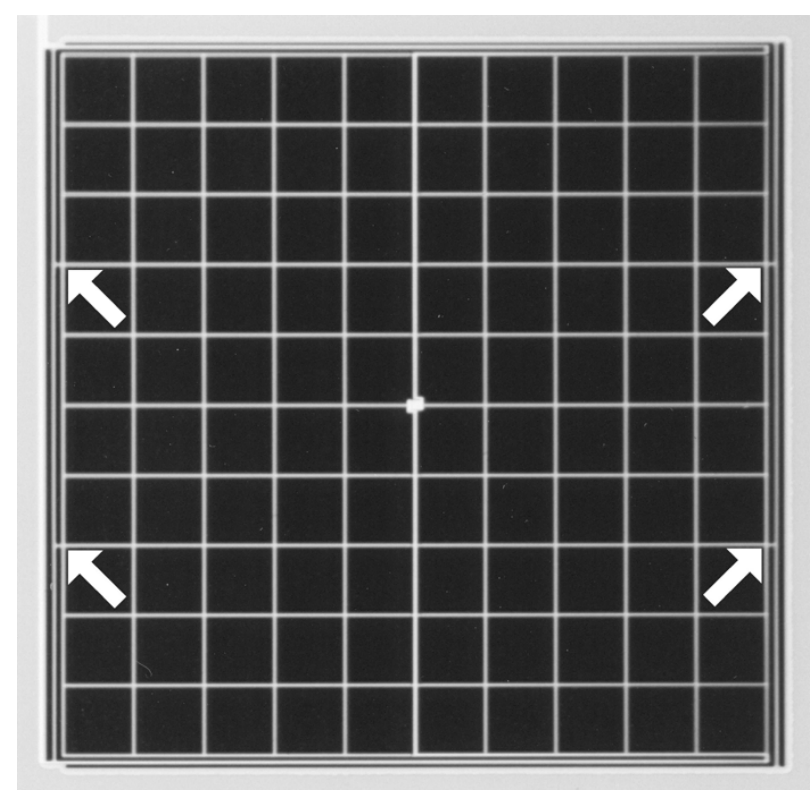

Fig. 3. Complete pixel including sensor and metallization for the array shown in Fig. 2. The grid is suspended at four points (arrows) by freestanding tensioned LSN strips which run along the left and right sides. Tests gave NEP $=2.3 \times 10^{-17} \mathrm{~W} \cdot \mathrm{Hz}^{-1 / 2}$ and $\tau=24 \mathrm{~ms}$ with $G=2.7 \times 10^{-11} \mathrm{~W} \cdot \mathrm{K}^{-1}$ at $304 \mathrm{mK}$.

pixel is shown in Fig. 3. The absorbing element is a square mesh of $1-\mu$ m-thick LSN several wavelengths across, which is metallized with gold to produce an average sheet resistance of $377 \Omega$ per square. A conducting backshort is located a distance $\lambda / 4$ behind the mesh. This mesh absorber is designed for low heat capacity and low cosmic ray cross section. It is supported at four points (arrows) by low thermal conductivity beams of LSN which lie along the edges to permit close packing of the pixels. The thermistor at the center of the mesh is made from a proximity-effect sandwich of $\mathrm{Al}$ and Ti to produce $T_{c} \sim 400 \mathrm{mK}$, which is appropriate for bolometers operated using ${ }^{3} \mathrm{He}$ sorption refrigerators. Fully superconducting leads will be used from the thermistors to the edges of the array by way of the beams and the dividing strips between pixels. Other designs for close-packed arrays use micromachining and folding [92] to bring the leads out in the third dimension. Alternatively, In bump bonds may be used to bring the leads to a second wafer which contains the wiring and the backshorts or even the SQUID multiplexer circuitry. Arrays of 1280 such bolometers are being developed for the SCUBA-2 instrument for the JCMT telescope [93], to be used in $2 \times 2$ mosaics.

Some of the most successful conventional submillimeter and millimeter wave bolometer systems use an array of close-packed conical horn anntenas in the focal plane. The bolometers, which are located at the small ends of the horns, are then separated sufficiently for easy support and wiring. The TES spiderweb bolometer structure [94] shown in Fig. 4, which has radial support legs, is appropriate for such systems. Arrays of such bolometers are being fabricated for ground-based surveys for clusters of galaxies using the Sunyaev-Zeldovich effect.
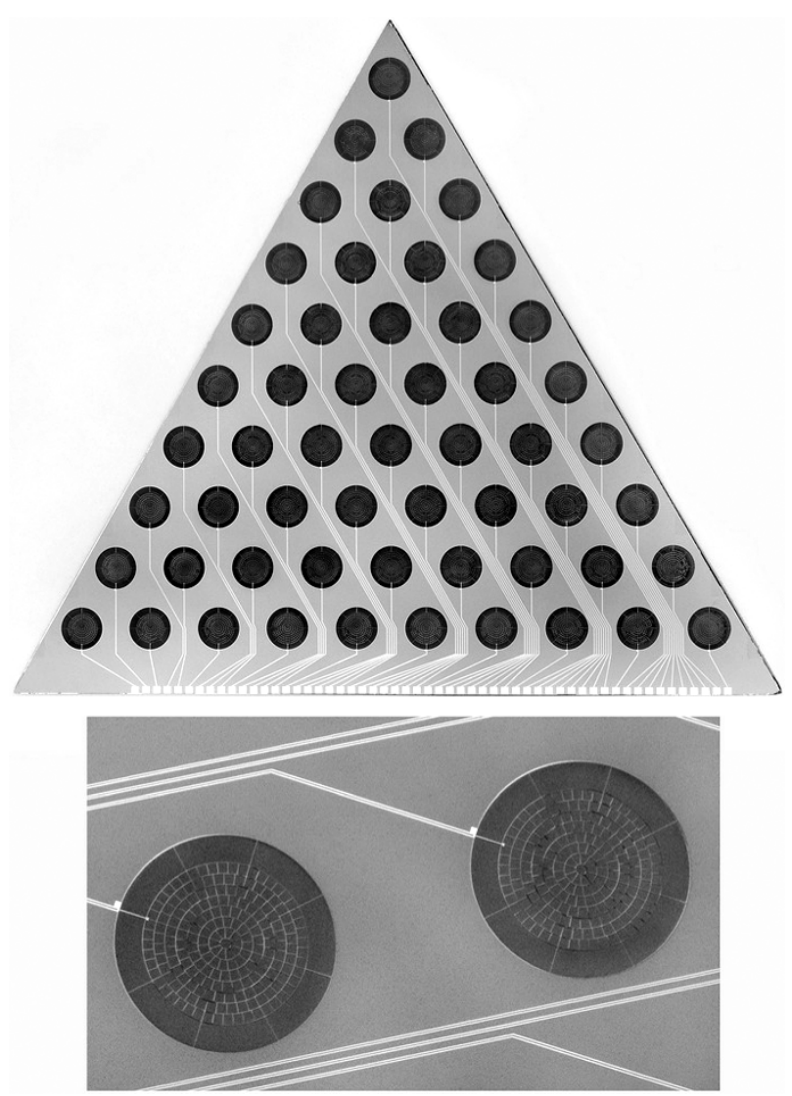

Fig. 4. Array of 55 TES spiderweb bolometers and closeup of bolometers. Six wedges of the type shown will be assembled to form a 330-element hexagonal horn-coupled array.

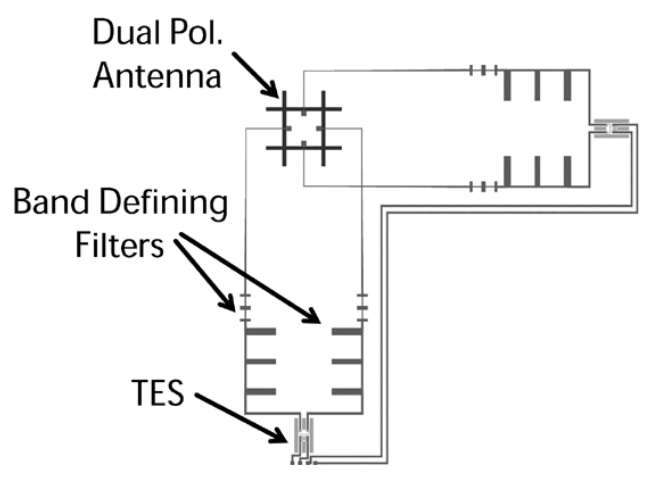

Fig. 5. Antenna for TES bolometers. A double slot dipole dual-polarization antenna is at the left. In-line band pass filters are at the top.

Interest is developing in the use of planar lithographed antennas to couple bolometers to a telescope [95]-[102] (see also Section IV-C and Figs. 12 and 13). This approach is very promising for $\mathrm{CMB}$ polarization measurements. Fig. 5 shows TES bolometers coupled by a dual-polarization double-slot dipole antenna [103], [104] in a Nb ground plane. Antennas of this type couple to the telescope optics by way of extended hemispherical Si lenses. Another approach is to combine the outputs of several antennas with the proper phases to produce a narrower antenna pattern which can couple directly to the 


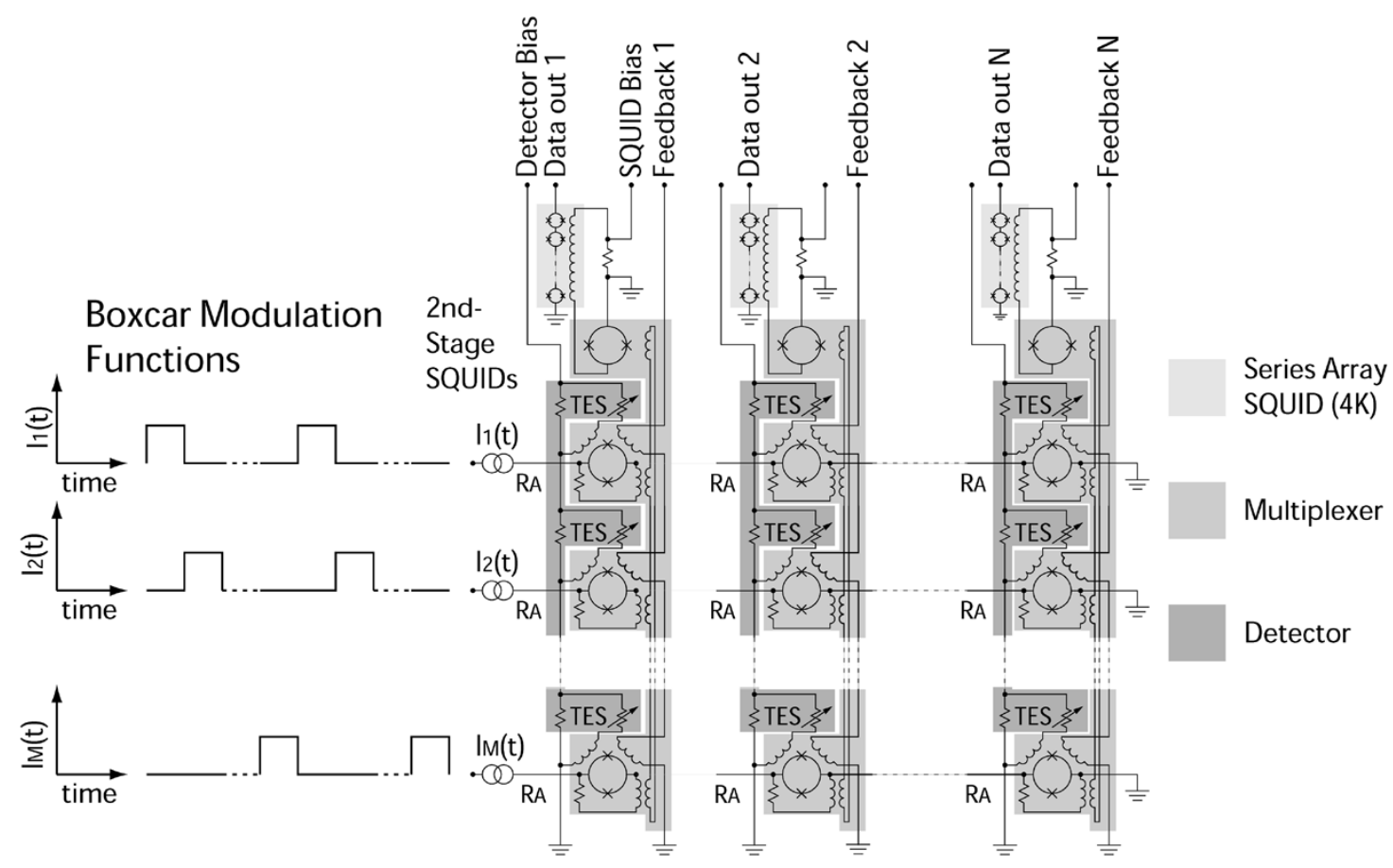

Fig. 6. NIST time-domain multiplexer. Each dc-biased TES detector (dark gray) is coupled to a normally closed SQUID switch (medium gray). The switches on a column are wired in series to an array SQUID output amplifier (light gray). When the boxcar control signal is positive, the corresponding row of switches opens, coupling the outputs of the TESs in the row to their output amplifiers.

telescope optics [105]-[107]; a prototype antenna of this type has already been demonstrated. In the device shown in Fig. 5, $\mathrm{Nb}$ microstrip lines couple the antennas to microstrip filters which are used to define the measured frequency band and then to small resistive terminations which form the absorbing elements of leg-isolated TES bolometers. Transmission line filters are far more compact than the metal mesh filters that are conventionally used with bolometers. It is hoped that they will also be more efficient. It seems possible to use a transmission line channelizer to obtain several bands from a single antenna. Much of the superconducting antenna, microstrip, and filter technology being explored for these bolometers has been previously developed for SIS heterodyne mixers at submillimeter and millimeter wavelengths and for picosecond Josephson digital logic. Antenna-coupled TES bolometer development is just beginning and many interesting ideas have yet to be tested.

The small area of these antenna terminations makes them compatible with an alternative TES device, the HEB [108]. A TES with a small area is deposited directly on the Si substrate without any legs for thermal isolation. At temperatures $\sim 0.1 \mathrm{~K}$, the weakness of the electron-phonon interaction impedes the flow of heat from the metal into the dielectric substrate and can provide values of thermal conductance $G$ that are appropriate for low-background astronomical bolometers. Techniques for further weakening the electron-phonon interaction are being explored [109]-[111] with the goal of producing bolometers with $\mathrm{NEP} \ll 10^{-18} \mathrm{~W} \cdot \mathrm{Hz}^{-1 / 2}$. At the other extreme, the use of very fast HEBs for heterodyne mixing is described in Section V.

\section{E. Output Multiplexing}

Instruments are now being developed that will have thousands of bolometers in a focal plane operated at temperatures below $1 \mathrm{~K}$; the most ambitious example is the SCUBA-2 instrument with $\sim 10^{4}$ pixels [93], [112]. Many difficult cryogenic problems arise in such systems. If, for example, a SQUID amplifier is required for each bolometer, there must be at least five leads from the cold stage for each pixel. Much effort is being made to produce an output multiplexer that will permit a column of $\sim 30$ bolometers to be read out through a single SQUID amplifier without any degradation in performance. Such multiplexers will be of great value in applications of other TES devices, including $\mathrm{X}$-ray calorimeters.

Two generic approaches are being explored. In the time-domain multiplexer, each bolometer is equipped with a switch activated by a control line. Signals from the column of bolometers are switched sequentially to the output amplifier. The number of bolometers $N$ that can be multiplexed is limited by the fact that each bolometer is read out for $1 / N$ th of the time. The noise margin of the output amplifier is, thus, degraded by a factor $N^{1 / 2}$. The National Institute of Standards and Technology (NIST) group [113]-[115] has developed a time domain multiplexer that uses SQUIDs as switches, as shown in Fig. 6. Several generations of this multiplexer have been successfully tested, and experience is being obtained with small systems at telescopes [28].

In the frequency-domain multiplexer being developed by groups at Berkeley [116]-[118] and elsewhere [119]-[121], 


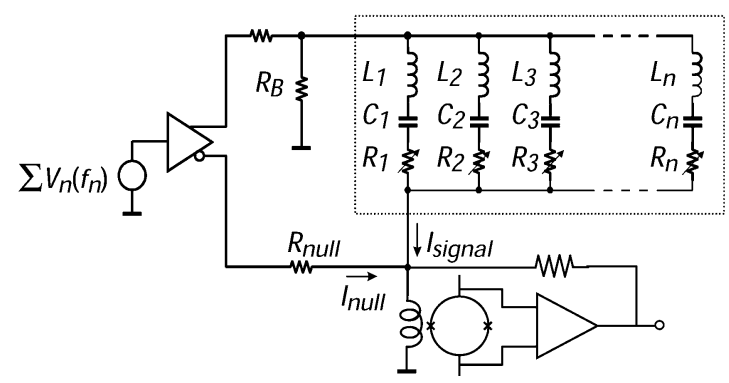

Fig. 7. Berkeley frequency-domain multiplexer. Each TES is ac biased at a separate frequency. $L C$ filters are used to select the bias from a comb of frequencies on a single bias line and to prevent build up of broad-band Johnson noise in the output from each TES. The currents through a row of detectors are summed at the input of an array SQUID amplifier. Out-of-phase signals from the bias source can be used to null the carrier frequencies from each TES to reduce the demands on the amplifier.

each bolometer in a line is ac biased at a different frequency. The bolometer currents are summed at the input of a series array SQUID amplifier, as shown in Fig. 7. Lock-in demodulators are used at ambient temperature to recover the bolometer signals. The limitation to the number of bolometers that can be multiplexed is set by the slew rate of the SQUID output amplifier. Since the optical signals are contained in the sidebands of the carrier frequencies, inputs from the bias source can be used to null the carriers at the input to the amplifier. Using this technique, it should be possible to multiplex $\sim 30$ bolometers without degradation of performance. The frequency-domain multiplexer is not as advanced as the time-domain system. Prototypes have been produced and are being tested.

\section{PAIR-BREAKIng AND TunNel Junction Detectors}

\section{A. Pair-Breaking Detectors}

An alternative approach to photon detection using superconductivity is to operate far below the transition temperature $T_{c}$. In this situation, most of the electrons are bound together into Cooper pairs [122]. Photons absorbed in the superconductor may break apart these Cooper pairs to produce single electrons, or, more accurately, quasi-particles, provided that the photon energy $h \nu$ exceeds the binding energy of the pair $2 \Delta=3.5 k_{\mathrm{B}} T_{c}$. This process is very analogous to the creation of an electron-hole pair in a semiconductor photodetector. One key advantage of this type of detector, which was recognized very early [123], is that the fundamental noise due to the random generation and recombination of thermal quasi-particles [124] decreases exponentially with temperature, as $\exp \left(-\Delta / k_{B} T\right)$. In contrast, bolometric detectors convert the incoming radiation into heat rather than quasiparticle excitations, and their sensitivity exhibits a slower power-law dependence with temperature. However, since the photoproduced quasi-particles are in a sea of Cooper pairs, one needs a method to measure their presence or to separate them out.

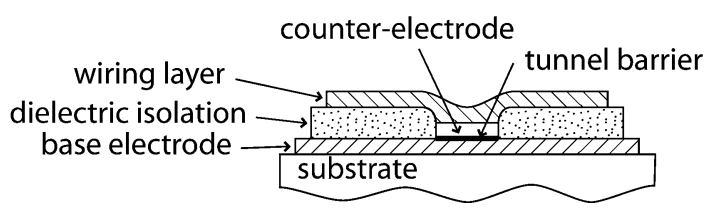

Fig. 8. A cross section of a typical SIS tunnel junction. Typical substrate materials on which the layers are deposited are high-resistivity silicon or sapphire for quasi-optical mixers or quartz for waveguide mixers. For a standard junction process, the base electrode is $\sim 200$-nm sputtered niobium, the tunnel barrier is made using a thin $(\sim 5-\mathrm{nm})$ sputtered aluminum layer which is either thermally oxidized $\left(\mathrm{Al}_{2} \mathrm{O}_{3}\right)$ or plasma nitridized (AlN), and the counterelectrode is $\sim 100$-nm sputtered niobium or reactively sputtered NbTiN. The entire SIS trilayer is deposited in a single deposition run. The junction area is then defined by photolithography or electron-beam lithography and reactive ion etching (RIE) of the counterelectrode, followed by deposition of the dielectric isolation layer $(\sim 200 \mathrm{~nm})$, which is typically thermally evaporated $\mathrm{SiO}$ or sputtered $\mathrm{SiO}_{2}$. The wiring layer provides the contact to the top of the counterelectrode, and is usually niobium for $\nu<700 \mathrm{GHz}$, and either NbTiN or a good-quality normal metal (e.g., $\mathrm{Al}$ or $\mathrm{Au})$ for higher frequencies.

\section{B. STJ Detectors}

The first suggestion for a pair-breaking detector, made over 40 years ago [123], involved tunnel junctions (see Fig. 8); these are generically called superconducting tunnel junction (STJ) detectors. In a rough sense, the tunnel junctions are used to filter out the Cooper pairs but let the quasi-particles pass. Single energetic particles or photons may break a large number of Cooper pairs into quasi-particles, thereby producing a current pulse flowing through the tunnel junction, whose amplitude indicates the energy of the event [125]-[136]. Alternatively, a steady flux of submillimeter photons absorbed in the superconductor will produce a dc current in the junction. Antenna-coupled versions (see Section IV-C) of this idea [137] could yield very sensitive detectors, perhaps in the $10^{-20} \mathrm{~W} \mathrm{~Hz}^{-1 / 2}$ range. Ideas have been proposed for multiplexed readout of tunnel junction detectors [138] using RF single-electron transistors (RF-SETs) [139].

\section{RF Kinetic Inductance Detectors}

The quasi-particles produced by pair-breaking photons may also be sensed by measuring the complex ac surface impedance of the superconductor. At finite frequencies, the surface impedance is nonzero and is in fact largely inductive; this is known as the kinetic inductance effect due to its connection with the kinetic energy of motion of the Cooper pairs. Furthermore, the surface impedance responds to changes in the quasi-particle population, even at very low temperatures $T \ll T_{c}$ [140], [141]. This opens up the possibility of making very simple detectors [142], [143], which consist of high- $Q$ thin-film microwave resonators, using coplanar waveguide transmission lines [144] for example. Photons absorbed in the superconductor change the resonance frequency, which can be sensitively monitored using an RF/microwave readout system. Such RF kinetic inductance detectors (RF-KIDs) have now been demonstrated [145], [146] for single-photon X-ray detection and have the great advantage of being easily frequency multiplexed. 


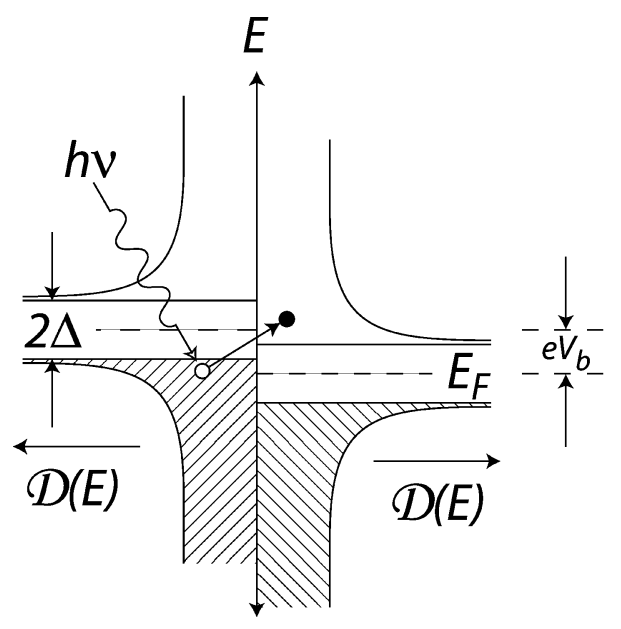

Fig. 9. Energy diagram for photon-assisted tunneling. The superconductors on the two sides of the tunnel junction are represented in the simplified "semiconductor" picture, in which the states below the energy gap are considered to be occupied, and those above the gap are empty. The curves indicate the electron density of states, $\mathcal{D}(E)$, which is zero inside the $2 \Delta$ gap region, and has square-root singularities at the gap edges. When a bias voltage $V_{b}$ is applied to the junction, there is a relative energy shift of $e V_{b}$ between the Fermi levels of the two superconductors, as illustrated. Electrons may only tunnel into unoccupied states at the same energy; thus, no current flows until $e V_{b}>2 \Delta$. However, if an ac voltage at frequency $\nu$ is applied, photons of energy $h \nu$ may assist the tunneling, which now may occur for $e V_{b}>2 \Delta-h \nu$, as depicted.

Large arrays of such detectors seem to be quite feasible. Another highly appealing aspect of RF-KIDs, especially from a systems engineering standpoint, is that almost all of the readout circuitry would be at room temperature, outside the cryostat, and could make use of the RF integrated circuits developed for wireless communications. Superconducting detectors with microwave readouts have been proposed in the past, but using nonresonant measurements of changes in the surface resistance [147], [148].

\section{SQUID Kinetic Inductance Detectors}

Another possibility is to use a dc SQUID to read out the changes in the kinetic inductance (SQUID-KIDs). This idea was originally proposed for bolometers using kinetic inductance thermometers [149]-[154]. For this mode of operation, the radiation is converted to heat, and so the temperature of the superconductor must be kept close to $T_{c}$, where the variation of the kinetic inductance with temperature is largest. As noted earlier, a significant advantage can be gained if the radiation is directly absorbed by the superconductor to produce quasi-particle excitations rather than heat; SQUID-KIDs using this $T \ll T_{c}$ mode of operation have been proposed [140], [155]. Multiplexed readouts of SQUID-KIDs should be possible, with approaches similar to those followed for TES bolometers, but have not been studied in any detail yet.

\section{E. SIS Photon Detectors}

As discussed in Section IV-B (see Fig. 9), superconducting (SIS) tunnel junctions may directly convert submillimeter photons to electrical current through the process of photon- assisted tunneling, with a responsivity approaching one electron per photon. This effect is widely used for heterodyne mixing, but may also be employed for direct detection. The sensitivity of this device is determined by the leakage current, which can be rather high for low-resistance junctions that are easily matched to antennas. Nonetheless, an NEP in the range $10^{-16} \mathrm{~W} \cdot \mathrm{Hz}^{-1 / 2}$ appears to be possible, and this device is actively being developed for astronomical use [156].

\section{F. SIN Junction Microbolometers}

An SIN tunnel junction differs from an SIS junction (see Fig. 8) in that one of the electrodes is a normal metal. In order for an electron to tunnel from the normal metal into the superconductor, it must have an energy above the Fermi level of at least $\Delta-e V_{b}$, where $\Delta$ is the gap parameter of the superconductor and $V_{b}$ is the junction bias voltage. Thus, the junction current probes the tail of the Fermi distribution of electrons in the normal metal, and consequently is exponentially sensitive to the electron temperature $T_{e}$, scaling as $\exp \left[-\left(\Delta-e V_{b}\right) / k_{B} T_{e}\right][157]$. An SIN junction is, therefore, a good thermometer for measuring the electron temperature in a normal metal. This property was exploited by Nahum and Martinis [158] to design a hot-electron microbolometer, in which the absorbed radiation heats the electrons in the normal metal, and the increase in $T_{e}$ is measured using the SIN junction. SIN microbolometers enjoy a negative feedback effect [158], [159], since the tunneling electrons also carry away heat from the normal metal. The theory of these devices has been developed in some detail [160], [161], experimental characterization of antenna-coupled detectors is ongoing [162], [163], and the use of a dc SQUID readout is being investigated [164].

\section{TunNel JunCtion (SIS) MiXerS}

\section{A. Introduction}

STJ mixers, also known as SIS mixers due to the superconductor/insulator/superconductor sandwich structure of the junctions (see Fig. 8), may be thought of as high-performance versions of the well-known semiconductor diode mixers. In diode mixers, the mixing process can be considered to be a classical effect, arising from the rectification of the RF waveform applied to the diode due to the nonlinear nature of the current-voltage $(I-V)$ characteristic of the diode. However, for SIS mixers, it is necessary to take a quantum-mechanical view of the device operation, in which the output current is considered to be the result of photon-assisted tunneling of electrons. The physics of this effect was demonstrated experimentally in the early 1960s by Dayem and Martin [165], and explained theoretically by Tien and Gordon [166]. The first demonstrations of SIS mixing were published [167], [168] in 1979, after the technology for making suitably small-area SIS junctions had been developed. Phillips and Woody [43] provided an interesting summary of early SIS work. A more comprehensive review of the field was given in 1985 by Tucker and Feldman [58]; this paper still serves as the fundamental reference for SIS mixers. Subsequent reviews [45], [50], [169]-[172] give summaries of more recent developments. 


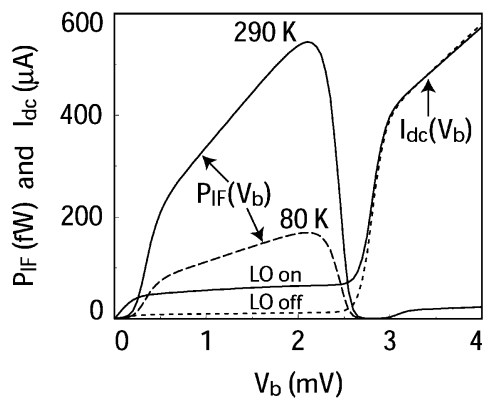

Fig. 10. Results of a detailed calculation of the operation of a 650-GHz SIS mixer [184], [185]. The lower curves are the dc $I-V$ curves of the tunnel junction with a 200-nW 650-GHz LO turned on (solid) and off (dashed). The unpumped $I-V$ curve shows the typical sudden turn-on of the tunneling current for bias voltages above the gap voltage, $V_{b}>2 \Delta / e \approx 2.8 \mathrm{mV}$. Above the knee voltage $\sim 3 \mathrm{mV}$, the $I-V$ curves are nearly linear, and the slope defines the "normal resistance" $R_{N}$. At $650 \mathrm{GHz}$, the photon voltage is $h \nu / e \approx 2.7 \mathrm{mV}$, so the first photon step extends nearly over the entire subgap region. The upper curves show the IF output power emerging in a 200-MHz bandwidth centered at $6 \mathrm{GHz}$, for the case that blackbody loads are placed at the mixer input with temperatures of $290 \mathrm{~K}$ (solid) and $80 \mathrm{~K}$ (dashed). The mixer noise temperature as a function of $V_{b}$ may be calculated from the ratio $Y=P_{\mathrm{IF}}(290 \mathrm{~K}) / P_{\mathrm{IF}}(80 \mathrm{~K})$.

\section{B. Basic Physics and Limitations}

The basic physics of photon-assisted tunneling is described in Figs. 9 and 10. In short, the existence of an energy gap $2 \Delta$ in the electronic density of states in a superconductor prevents electrons from tunneling across the junction until the applied bias voltage $V_{b}$ provides enough energy to the tunneling electrons to overcome the gap energy. Thus, the current turns on sharply for $V_{b}>2 \Delta / e$, as shown in Fig. 10. However, when an RF field at frequency $\nu$ is applied, photon-assisted tunneling is possible, which now can occur for $V_{b}>2 \Delta / e-h \nu / e$.

Thus, SIS junctions behave very much like optical or IR photodiodes, in which the output current consists of one electron per photon absorbed. Indeed, this simple intuitive picture is able to explain the basics of SIS design and operation [43], [170], [173]-[175]. In this picture, the key figure of merit is the quantum efficiency $\eta<1$, which describes the overall probability for an incoming photon to be absorbed by the SIS junction to produce a tunneling electron. By careful design of the coupling structure, and especially paying attention to the impedance match to the SIS junction, the ideal limit $\eta \rightarrow 1$ may be approached. For a given value of quantum efficiency, the best noise temperature that may be obtained is $k_{B} T_{n} \geq h \nu / \eta$ [single sideband (SSB)]. The photodiode picture also explains one of the principal frequency limitations of SIS mixers: for $h \nu>4 \Delta$, the photon energy is sufficiently large to allow photon-assisted tunneling in the reverse direction. For all-Nb SIS junctions, the limit is $\nu \leq 1.4 \mathrm{THz}$; operation to $1.6 \mathrm{THz}$ should be possible if $\mathrm{NbN}$ or $\mathrm{NbTiN}$ is used for at least one junction electrode.

In order to go beyond the photodiode picture, to be able to perform detailed simulation and optimization of mixer circuits, one needs a more comprehensive theory. This theory was provided by Tucker [58], [176]-[178], who extended the Tien-Gordon analysis and tunnel junction noise theory [179] to provide a complete description of the signal and noise properties of tunnel junctions used as mixers. Tucker's theory is a semiclassical theory, in which the junction is treated quantum mechanically while the radiation field is treated classically. The extension to a fully quantum approach is not difficult [173] and shows that the sensitivity of an SIS receiver system can approach [180] but never be better than the quantum limit: $T_{n} \geq h \nu / k$ (SSB). Detailed design calculations using the Tucker theory require significant computation, including solution of the nonlinear problem of finding the LO waveform, which is typically done using harmonic-balance techniques [181], [182]. Stability issues [183] are also very important. Sophisticated software packages are available to perform these computations for multijunction circuits, including the simulation of the superconducting RF circuitry [184], [185]; see Fig. 10.

\section{Design Approaches}

A typical tunnel junction size is $\sim 1 \mu \mathrm{m}$. Because this is so much smaller than the wavelength being received, an antenna and associated coupling circuitry are needed to bring the radiation to the junction. There are two major ways to accomplish this task: waveguide coupling and quasi-optical coupling. The more traditional microwave-engineering approach is waveguide coupling, in which the radiation is first collected by a horn into a single-mode waveguide, typically a rectangular guide. One then needs a transition or "probe" which couples radiation from the waveguide onto a lithographed thin-film transmission line on the SIS chip itself. There are numerous examples of this approach [45], [186]-[188]; see Fig. 11. Using modern three-dimensional electromagnetic simulation software, it is now possible to design probes which offer excellent performance over an entire waveguide band [189]. One major complication of the waveguide approach is that the mixer chip must be very narrow, and must be fabricated on an ultrathin substrate. Modern micromachining techniques [190]-[192] are helpful in this regard (see Figs. 11 and 15), and mixer assembly may be simplified through the integration of beam leads on the SIS chip [192]-[194].

The other major approach is quasi-optical coupling, shown in Fig. 12, in which case one omits the intermediate step of collecting the radiation into a waveguide, and instead uses a lithographed antenna on the SIS chip itself. Such mixers are substantially simpler to fabricate and may be produced using thick substrates. The first such SIS mixer [195] used a bow-tie antenna, which is broadband but suffers from a nonideal beam pattern [196]. Subsequent experimental and theoretical work [197]-[207] has shown that a 90\% coupling efficiency may be achieved using optimized antennas and lenses. Dual-polarization designs are also possible [103], [208], [209]. Planar antenna calculations are typically carried out using rigorous moment-method techiques, and the effect of the lens is evaluated using ray-tracing and diffraction calculations, sometimes including multiple internal reflections. 


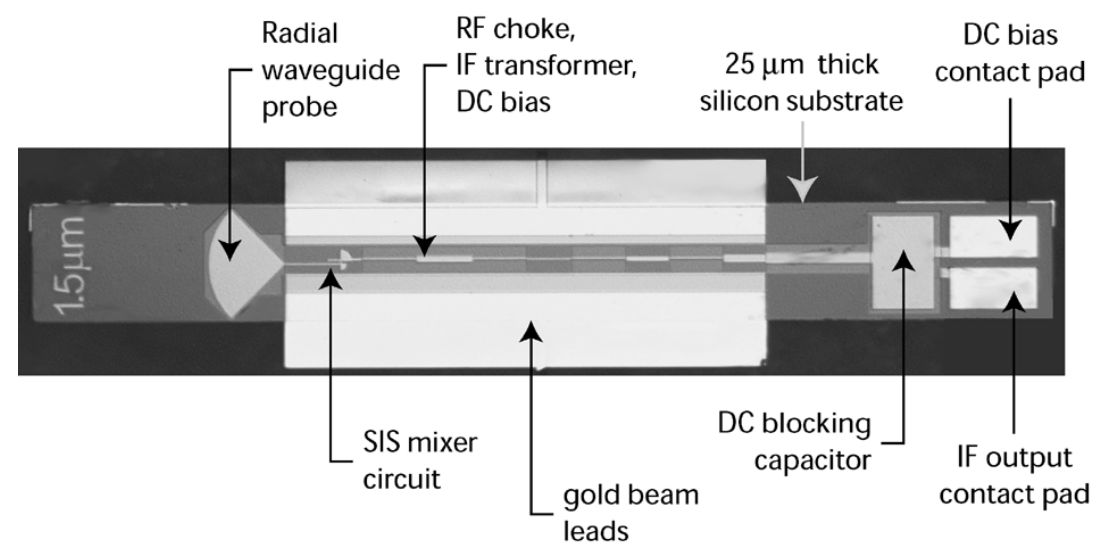

Fig. 11. Image of a waveguide SIS mixer chip designed for the 200-300 GHz band [247]. The chip is approximately $2 \mathrm{~mm}$ long and $0.24 \mathrm{~mm}$ wide, and was fabricated at JPL [272] on 25- $\mu$ m-thick silicon, through a novel JPL-developed process utilizing silicon-on-insulator (SOI) bonded wafers, in which the $25-\mu \mathrm{m}$ silicon layer is mechanically supported by a much thicker "handle" layer throughout most of the processing. The 1- $\mu \mathrm{m}$-thick gold beam leads extend beyond the edges of the substrate and are used to make a good electrical "ground" contact to the metal waveguide block, facilitating mixer assembly. This design uses a broadband radial waveguide probe [189], [273], a novel distributed/lumped-element SIS matching circuit with a series tuning inductance, a Nb/AlN/Nb SIS junction, and a broadband CPW/microstrip RF choke that simultaneously serves as a 4-20 GHz IF output transformer. Fig. 15 shows another example of a chip fabricated on ultrathin silicon-in that case, a $585-\mathrm{GHz}$ HEB mixer.

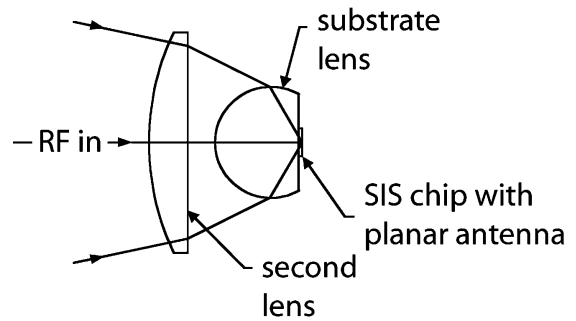

Fig. 12. Schematic diagram of the "reverse-microscope" quasi-optical coupling approach pioneered by Rutledge and Muha [274]. Because planar antennas on dielectric substrates tend to have wide beam patterns and receive radiation preferentially from the dielectric side, a hyperhemispherical or elliptical substrate lens is used to convert the incoming radiation into a wide-angle converging beam inside the dielectric, coming to a focus on the antenna, thereby matching the antenna receiving pattern. An antireflection coating on the lens may be used to improve the efficiency [201].

\section{Tuning Circuits, Materials Properties, and Terahertz Operation}

One of the major challenges in SIS mixer design is providing an impedance match to the tunnel junction, which is highly capacitive, since it is a parallel-plate structure with two electrodes that are separated by only $\sim 1 \mathrm{~nm}$ of insulation. Typical values of the junction specific capacitance are in the range $60-100 \mathrm{fF} \mu \mathrm{m}^{-2}$, depending on the materials used, current density, etc. Thus, for a typical $1-\mu \mathrm{m}^{2}$ junction, $1 / \omega C \sim 4 \Omega$ at $500 \mathrm{GHz}$-quite far from the typical $\sim 50-\Omega$ impedance levels for RF circuits. As a consequence, on-chip inductive tuning circuits are needed to compensate for the junction capacitance. The tuning inductor may be either a parallel [186], [210], [211] or series [212] element, or be placed in between two SIS junctions [213]-[215]; see Fig. 13. Typically, the tuning inductance is a short section of thin-film superconducting microstrip line [216]-[218]. For

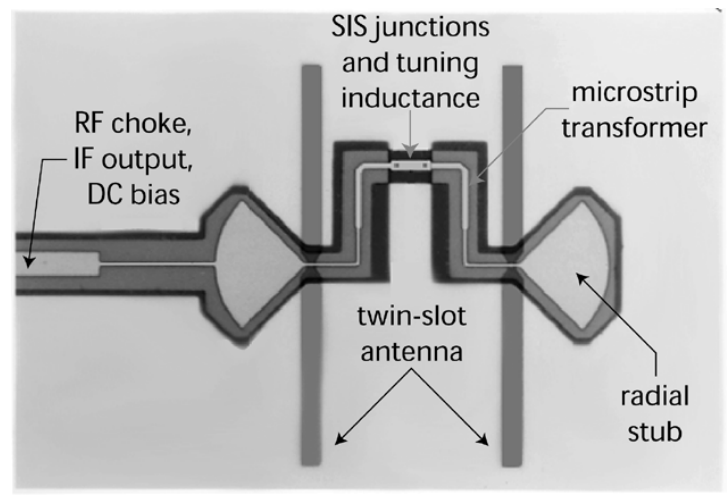

Fig. 13. Image of the central region of a quasi-optical SIS mixer chip with a twin-slot antenna [220]. The radial stubs serve as RF short circuits and couple the radiation received by the slots into the thin-film $\mathrm{Nb} / \mathrm{SiO} / \mathrm{Nb}$ superconducting microstrip circuitry. This chip uses two $\mathrm{Nb} / \mathrm{Al}_{2} \mathrm{O}_{3} / \mathrm{Nb}$ SIS junctions [275]; the short microstrip section in between the junctions provides the tuning inductance needed to compensate the junction capacitance [214].

all of these cases, the RF bandwidth $\Delta \nu$ of the mixer is fundamentally limited by the $R C$ product of the SIS junctions [219], to about $\Delta \nu \sim 100 \mathrm{GHz}$ for $\mathrm{Nb} / \mathrm{Al}$-oxide/ $\mathrm{Nb}$ junctions [220] and increasing to $\Delta \nu \sim 300 \mathrm{GHz}$ for devices with AlN barriers [221]. This bandwidth limit may be circumvented using distributed circuit techniques [222]-[224], at the expense of fabrication and/or design complexity.

One of the reasons that millimeter-wave SIS mixers are so sensitive is that the tuning inductance has very little loss. However, above $700 \mathrm{GHz}$, tuning inductors made with the most commonly used superconductor (niobium) become lossy [225] because photons have enough energy to break Cooper pairs $(h \nu>2 \Delta)$, and the mixer performance degrades rapidly. Various approaches have been used to mitigate this problem [226], including the use of normal 


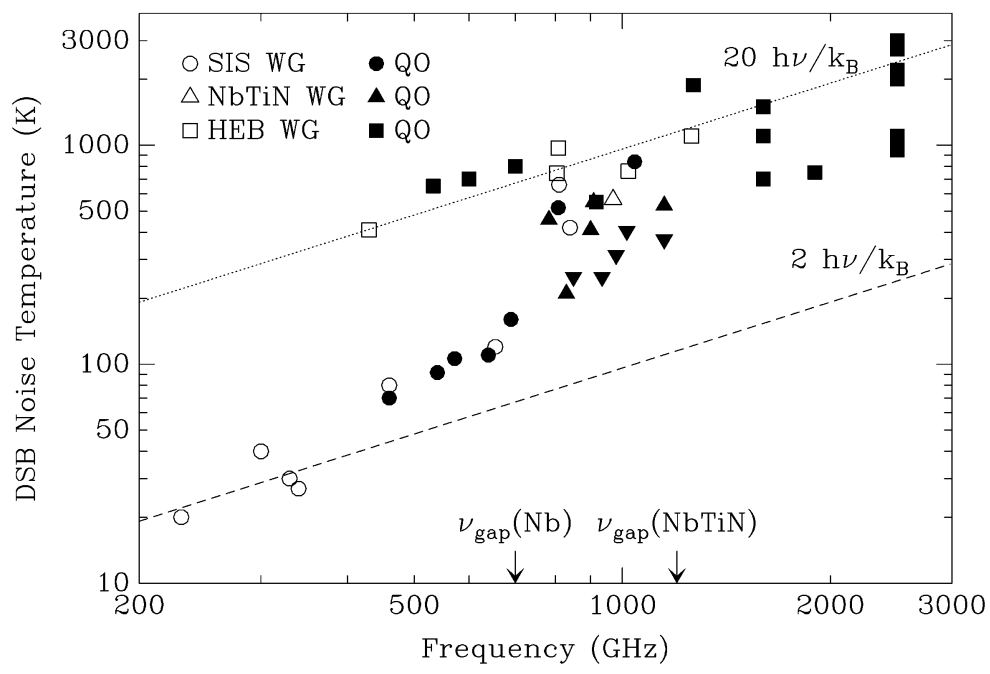

Fig. 14. Selected receiver sensitivities, given as the uncorrected double-sideband (DSB) noise temperature. The lines labeled $2 h \nu / k_{B}$ and $20 h \nu / k_{B}$ correspond to DSB noise temperatures of 0.1 and $1 \mathrm{~K} / \mathrm{GHz}$, respectively. SIS results obtained using standard niobium technology are shown as circles, either open (waveguide) or filled (quasi-optical). SIS results using $\mathrm{NbN}$ or NbTiN materials in the tuning circuit or junction electrodes are similarly given by upward-pointing triangles. The downward-pointing triangles represent $\mathrm{NbN} / \mathrm{NbTiN}$ results corrected for the LO injection beamsplitter loss, which can be significant when using low-power tunable terahertz LO sources. HEB results are given as open (waveguide) or filled (quasi-optical) squares. The SIS results are taken from [190], [220], [222], [227], [231], [232], [234], [235], [237], [238], [276]-[284]. The HEB data are from [236], [285]-[296].

metal circuits [227], [228], higher gap superconductors such as $\mathrm{NbN}$ or $\mathrm{NbTiN}$ [229]-[237], and very high current density AlN-barrier junctions [221], [233], [238], [239]. At present, SIS mixers have been successfully operated at frequencies up to $1.25 \mathrm{THz}$ - the latest result for the Herschel/HIFI $1.2-\mathrm{THz}$ SIS mixer is $530 \mathrm{~K}$ (DSB) uncorrected, and below $400 \mathrm{~K}$ after correction for the LO beamsplitter [280]. The upper frequency limit using currently available technology is around $1.6 \mathrm{THz}$.

\section{E. Performance}

Fig. 14 shows a plot of selected receiver noise temperature results. Below $400 \mathrm{GHz}$, the best results are just a few times the quantum limit, dropping below $0.1 \mathrm{~K} / \mathrm{GHz}$. Above $500 \mathrm{GHz}$, quasi-optical receivers offer very strong competition to waveguide systems.

\section{F. New Directions}

Now that millimeter-wave SIS technology has reached a fairly mature state, the emphasis has shifted toward receivers that are more capable, easier to operate, and easier to assemble, particularly in response to the needs of projects such as ALMA [56], [240]. Development goals include wide RF bandwidth with fixed-tuned operation, wide IF bandwidth, dual polarization, balanced mixers, sideband separation, and beam-lead contacts [19], [192]-[194], [208], [209], [241]-[251]. The ALMA memo series [252] is a particularly valuable source of technical information for this area, describing the latest progress being made by the numerous institutions involved in ALMA development. The Space Terahertz Technology conferences [253] also capture much of the latest work.

\section{Hot-EleCtron BolOMETER MiXers}

HEB mixers using semiconductors were invented in the early 1970s [254] and played an important role in early submillimeter astronomy [43], but were superceded by SIS mixers by the early 1990s. However, the development of superconducting versions [255], [256] of this basic concept has led to the most sensitive mixers at frequencies in the terahertz region that are beyond the reach of SIS mixers. HEB receiver systems are just now starting to be used for astronomy [257].

In principle, HEB mixers are quite similar to the transition-edge bolometers described in Section II. A film is biased near its superconducting transition, so that its resistance is strongly influenced by the small temperature changes caused by the absorption of radiation. The main difference between HEB mixers and ordinary bolometers is the speed of their response: HEB mixers should be fast enough to allow $\mathrm{GHz}$ output (IF) bandwidths. High speed is achieved by allowing the radiation power to be directly absorbed by the electrons in the superconductor, rather than using a separate radiation absorber and allowing the energy to flow to the superconducting TES via phonons, as ordinary bolometers do. When a photon is absorbed in a metal, a single electron initially receives the photon energy $h \nu$. This energy is rapidly shared with other electrons, producing a slight increase in the electron temperature. The electron temperature subsequently relaxes to the bath temperature, usually through the emission of phonons.

An HEB takes advantage of this effect: near $T_{c}$, the superconductor's resistance is sensitive to the electron temperature. The thermal relaxation time $\tau$ of the electrons can be made fast by choosing a material with a large electronphonon interaction, such as $\mathrm{NbN}$ [256], [258], [259], and 


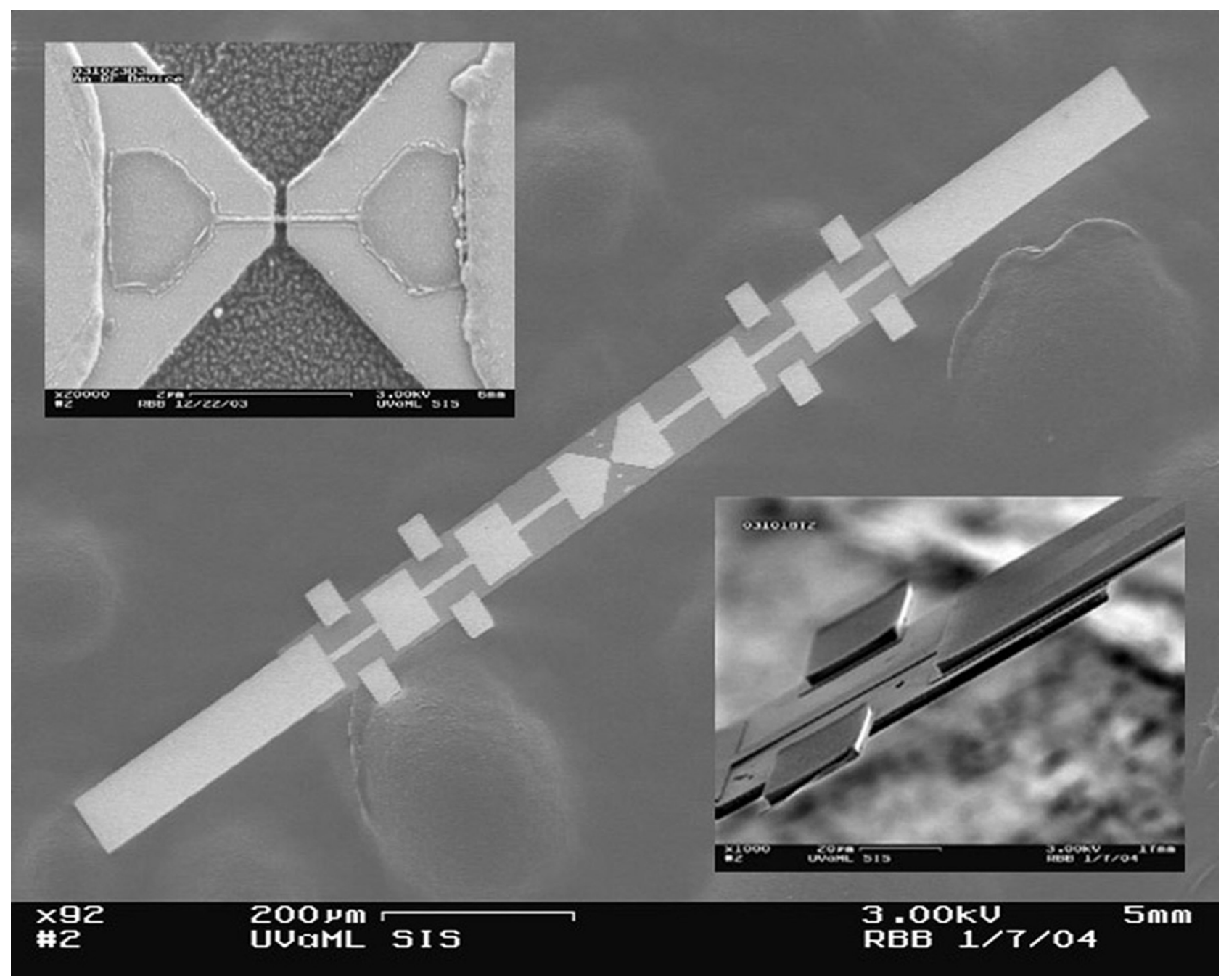

Fig. 15. Images of a 585-GHz diffusion-cooled HEB mixer chip for a waveguide mount, fabricated at the University of Virginia [192] using an ultrathin silicon substrate. The dimensions of the HEB bridge are $150 \mathrm{~nm}$ long by $75 \mathrm{~nm}$ wide; the chip itself is $800 \mu \mathrm{m}$ long, $78 \mu \mathrm{m}$ wide, and only $3 \mu \mathrm{m}$ thick. Protruding from the sides and ends of the chip are $2-\mu \mathrm{m}$-thick gold beam leads, which provide electrical and thermal contact to the waveguide block, as well as mechanical support for the chip.

using very thin films so that the phonons can escape into the substrate before being reabsorbed by the electrons. Alternatively, $\tau$ can be made rapid using electron outdiffusion [260]. For a fixed $\tau$, the heat capacity $C$ sets the required LO power and can be minimized by using a very small volume $\left(<10^{-2} \mu \mathrm{m}^{3}\right)$ of a superconducting film.

The theory of these devices is still under development, and typically invokes a "hot-spot" resistive region in the center, whose size responds to changes in the applied power [261]-[266]. Coupling to the device is very straightforward, since the RF impedance is essentially resistive, and can be done either with waveguide [191], [192] (see Fig. 15) or quasi-optical techniques as described in Section IV-C.

Both phonon-cooled and diffusion-cooled devices have been operated at $2.5 \mathrm{THz}$ and above, with IF bandwidths of several gigahertz. Selected results are shown in Fig. 14. The performance of these devices has been steadily improving [256] such that the best results now drop substantially below $1 \mathrm{~K} / \mathrm{GHz}$. The phonon-cooled $\mathrm{NbN}$ devices generally outperform the diffusion-cooled type at present, although improvements to the latter type may be possible [267]-[270]. Most of the HEB measurements at higher frequencies are performed using far-IR gas lasers, which have high output power at discrete frequencies, allowing the use of very weakly reflecting beamsplitters for LO injection. However, the LO power requirements for HEB mixers are quite low, and it should be possible to drive them using continuously tunable solid-state multiplier chains. An early experiment [271] at $1.5 \mathrm{THz}$ using a waveguide $\mathrm{NbN}$ mixer showed that this is indeed possible, achieving a DSB noise temperature around $1500 \mathrm{~K}$ with $\sim 1-\mu \mathrm{W}$ LO power.

\section{SUMMARY}

The millimeter through far-IR wavelength bands have enormous potential for scientific discovery, allowing the study of the birth of planets, stars, galaxies, and even the universe itself at $\sim 10^{-38} \mathrm{~s}$. Superconducting detectors and mixers are proving to be essential for realizing this potential. A whirlwind of innovation has swept the field during the 
last decade, yielding a wide variety of promising detector and mixer types, as well as providing breakthroughs in multiplexing for large arrays. Numerous techniques developed for more mature technologies (e.g., SIS mixers) are being adapted for use with newer devices (e.g., TES bolometers). One can hope that this flurry of innovation will lead to instruments that are powerful enough to achieve the scientific goals that we see on the horizon.

\section{REFERENCES}

[1] P. de Bernardis, P. Ade, J. Bock, J. Bond, J. Borrill, A. Boscaleri, K. Coble, B. Crill, G. De Gasperis, P. Farese, P. Ferreira, K. Ganga, M. Giacometti, E. Hivon, V. Hristov, A. Iacoangeli, A. Jaffe, A. Lange, L. Martinis, S. Masi, P. Mason, P. Mauskopf, A. Melchiorri, L. Miglio, T. Montroy, C. Netterfield, E. Pascale, F. Piacentini, D. Pogosyan, S. Prunet, S. Rao, G. Romeo, J. Ruhl, F. Scaramuzzi, D. Sforna, and N. Vittorio, "A flat universe from high-resolution maps of the cosmic microwave background radiation," Nature, vol. 404, no. 6781, pp. 955-959, 2000.

[2] S. Hanany, P. Ade, A. Balbi, J. Bock, J. Borrill, A. Boscaleri, P. De Bernardis, P. Ferreira, V. Hristov, A. Jaffe, A. Lange, A. Lee, P. Mauskopf, C. Netterfield, S. Oh, E. Pascale, B. Rabii, P. Richards, G. Smoot, R. Stompor, C. Winant, and J. Wu, "MAXIMA-1: A measurement of the cosmic microwave background anisotropy on angular scales of 10'-5 degrees," Astrophys. J., vol. 545, no. 1, pp. L5-L9, 2000.

[3] N. Halverson, E. Leitch, C. Pryke, J. Kovac, J. Carlstrom, W. Holzapfel, M. Dragovan, J. Cartwright, B. Mason, S. Padin, T. Pearson, A. Readhead, and M. Shepherd, "Degree angular scale interferometer first results: A measurement of the cosmic microwave background angular power spectrum," Astrophys. J., vol. 568, no. 1, pp. 38-45, 2002.

[4] D. Spergel, L. Verde, H. Peiris, E. Komatsu, M. Nolta, C. Bennett, M. Halpern, G. Hinshaw, N. Jarosik, A. Kogut, M. Limon, S. Meyer, L. Page, G. Tucker, J. Weiland, E. Wollack, and E. Wright, "Firstyear Wilkinson microwave anisotropy probe (WMAP) observations: Determination of cosmological parameters," Astrophys. J. (Suppl.), vol. 148, no. 1, pp. 175-194, 2003.

[5] M. Kamionkowski, A. Kosowsky, and A. Stebbins, "A probe of primordial gravity waves and vorticity," Phys. Rev. Lett., vol. 78, pp. 2058-2061, Mar. 1997.

[6] U. Seljak and M. Zaldarriaga, "Signature of gravity waves in the polarization of the microwave background," Phys. Rev. Lett., vol. 78, pp. 2054-2057, Mar. 1997.

[7] W. Hu and M. White, "A CMB polarization primer," New Astron., vol. 2, pp. 323-344, Sept. 1997.

[8] M. Kamionkowski and A. Kosowsky, "Detectability of inflationary gravitational waves with microwave background polarization," Phys. Rev. D, vol. 57, pp. 685-691, Jan. 1998.

[9] J. E. Carlstrom, G. P. Holder, and E. D. Reese, "Cosmology with the Sunyaev-Zel'dovich effect," Annu. Rev. Astron. Astrophys., vol. 40, pp. 643-680, 2002.

[10] I. Smail, R. J. Ivison, and A. W. Blain, "A deep submillimeter survey of lensing clusters: A new window on galaxy formation and evolution," Astrophys. J., vol. 490, pp. L5-L8, 1997.

[11] A. Barger, L. Cowie, D. Sanders, E. Fulton, Y. Taniguchi, Y. Sato, K. Kawara, and H. Okuda, "Submillimeter-wavelength detection of dusty star-forming galaxies at high redshift," Nature, vol. 394, no. 6690 , pp. $248-251,1998$.

[12] D. Hughes, S. Serjeant, J. Dunlop, M. Rowan-Robinson, A. Blain, R. Mann, R. Ivison, J. Peacock, A. Efstathiou, W. Gear, S. Oliver, A. Lawrence, M. Longair, P. Goldschmidt, and T. Jenness, "High-redshift star formation in the Hubble Deep Field revealed by a submillimeter-wavelength survey," Nature, vol. 394, no. 6690, pp. 241-247, 1998.

[13] A. W. Blain, I. Smail, R. J. Ivison, J.-P. Kneib, and D. T. Frayer, "Submillimeter galaxies," Phys. Rep., vol. 369, pp. 111-176, 2002.

[14] S. C. Chapman, A. W. Blain, R. J. Ivison, and I. R. Smail, "A median redshift of 2.4 for galaxies bright at submillimeter wavelengths," $\mathrm{Na}$ ture, vol. 422, pp. 695-698, Apr. 2003.
[15] J.-L. Puget, A. Abergel, J.-P. Bernard, F. Boulanger, W. B. Burton, F.-X. Desert, and D. Hartmann, "Tentative detection of a cosmic farinfrared background with COBE," Astron. Astrophys., vol. 308, pp. L5-L8, Apr. 1996.

[16] D. J. Fixsen, E. Dwek, J. C. Mather, C. L. Bennett, and R. A. Shafer, "The spectrum of the extragalactic far-infrared background from the COBE FIRAS observations," Astrophys. J., vol. 508, pp. 123-128, Nov. 1998.

[17] M. G. Hauser, R. G. Arendt, T. Kelsall, E. Dwek, N. Odegard, J. L. Weiland, H. T. Freudenreich, W. T. Reach, R. F. Silverberg, S. H. Moseley, Y. C. Pei, P. Lubin, J. C. Mather, R. A. Shafer, G. F. Smoot, R. Weiss, D. T. Wilkinson, and E. L. Wright, "The COBE diffuse infrared background experiment search for the cosmic infrared background. I. Limits and detections," Astrophys. J., vol. 508, pp. 25-43, Nov. 1998.

[18] P. M. Harvey, G. H. Rieke, D. F. Lester, and D. J. Benford, "Single aperture far-infrared observatory (SAFIR)," Proc. SPIE, IR Space Telescopes Instrum., vol. 4850, pp. 1097-1108, Mar. 2003.

[19] J. C. Webber and M. W. Pospieszalski, "Microwave instrumentation for radio astronomy," IEEE Trans. Microwave Theory Tech., vol. 50, pp. 986-995, Mar. 2002.

[20] P. Richards, "Bolometers for infrared and millimeter waves," J. Appl. Phys., vol. 76, no. 1, pp. 1-24, July 1994.

[21] A. Turner, J. Bock, J. Beeman, J. Glenn, P. Hargrave, V. Hristov, H. Nguyen, F. Rahman, S. Sethuraman, and A. Woodcraft, "Silicon nitride micromesh bolometer array for submillimeter astrophysics," Appl. Opt., vol. 40, no. 28, pp. 4921-4932, 2001.

[22] C. D. Dowell, J. E. Groseth, T. G. Phillips, C. A. Allen, S. R. Babu, M. D. Jhabvala, S. H. Moseley, and G. M. Voellmer, "The $12 \times 32$ pop-up bolometer array for the SHARC II camera," in Proc. Far-IR, Sub-MM, and MM Detector Workshop, vol. NASA/CP-2003-211 408, J. Wolf, J. Farhoomand, and C. R. McCreight, Eds., 2003, pp. 251-254.

[23] G. B. Heim, M. L. Henderson, K. I. Macfeely, T. J. McMahon, D. Michika, R. J. Pearson, G. H. Rieke, J. P. Schwenker, D. W. Strecker, C. L. Thompson, R. M. Warden, D. A. Wilson, and E. T. Young, "Multiband imaging photometer for SIRTF," Proc. SPIE, Space Telescopes Instrum. V, vol. 3356, pp. 985-1000, Aug. 1998.

[24] P. L. Richards, "Bolometric detectors for space astrophysics," in Proc. Far-IR, Sub-MM, and MM Detector Workshop, vol. NASA/CP-2003-211 408, J. Wolf, J. Farhoomand, and C. R. McCreight, Eds., 2003, pp. 219-223.

[25] T. G. Phillips and J. Keene, "Submillimeter astronomy," Proc. IEEE, vol. 80, pp. 1662-1678, Nov. 1992.

[26] C. M. Bradford, B. J. Naylor, J. Zmuidzinas, J. J. Bock, J. Gromke, H. Nguyen, M. Dragovan, M. Yun, L. Earle, J. Glenn, H. Matsuhara, P. A. R. Ade, and L. Duband, "WaFIRS: A waveguide far-IR spectrometer: Enabling spectroscopy of high- $z$ galaxies in the far-IR and submillimeter," Proc. SPIE, IR Space Telescopes Instrum., vol. 4850, pp. 1137-1148, Mar. 2003.

[27] T. Nikola, S. Hailey-Dunsheath, G. J. Stacey, D. J. Benford, S. H. Moseley, and J. G. Staguhn, "ZEUS: A submillimeter grating spectrometer for exploring distant galaxies," Proc. SPIE, Millimeter Submillimeter Detectors Astronomy, vol. 4855, pp. 88-99, Feb. 2003.

[28] J. G. Staguhn, D. J. Benford, F. Pajot, T. J. Ames, J. A. Chervenak, E. N. Grossman, K. D. Irwin, B. Maffei, S. H. Moseley, T. G. Phillips, C. D. Reintsema, C. Rioux, R. A. Shafer, and G. M. Voellmer, "Astronomical demonstration of superconducting bolometer arrays," Proc. SPIE, Millimeter Submillimeter Detectors Astronomy, vol. 4855, pp. 100-107, Feb. 2003.

[29] A. Poglitsch, C. Waelkens, and N. Geis, "The photodetector array camera and spectrometer (PACS) for the Herschel Space Observatory," Proc. SPIE, IR Space Telescopes Instrum., vol. 4850, pp. 662-673, Mar. 2003.

[30] C. M. Bradford, G. J. Stacey, M. R. Swain, T. Nikola, A. D. Bolatto, J. M. Jackson, M. L. Savage, J. A. Davidson, and P. A. R. Ade, "SPIFI: A direct-detection imaging spectrometer for submillimeter wavelengths," Appl. Opt., vol. 41, pp. 2561-2574, May 2002.

[31] T. de Graauw, N. D. Whyborn, H. van de Stadt, G. Beaudin, D. A. Beintema, V. Belitsky, P. Cais, E. Caux, M. Gheudin, A. Cros, P. de Groene, A. Emrich, N. R. Erickson, T. C. Gaier, and J. D. Gallego-Puyol, "Heterodyne instrument for FIRST (HIFI): Preliminary design," Proc. SPIE, Adv. Technol. MMW, Radio, Terahertz Telescopes, vol. 3357, pp. 336-347, July 1998.

[32] M. L. Edgar and J. Zmuidzinas, "CASIMIR: A submillimeter heterodyne spectrometer for SOFIA," Proc. SPIE, Airborne Telescope Syst., vol. 4014, pp. 31-42, June 2000. 
[33] P. Siegel, "Terahertz technology," IEEE Trans. Microwave Theory Tech., vol. 50, pp. 910-928, Mar. 2002.

[34] R. Guesten, I. Camara, P. Hartogh, H. Huebers, U. U. Graf, K. Jacobs, C. Kasemann, H. Roeser, R. T. Schieder, G. Schnieder, O. Sievertz, J. Stutzki, G. Villanueva, A. Wagner, P. van der Wal, and A. Wunsch, "GREAT: The german receiver for astronomy at terahertz frequencies," Proc. SPIE, Airborne Telescope Syst. II, vol. 4857, pp. 56-61, Feb. 2003.

[35] J. C. Pearson, I. Mehdi, E. Schlecht, F. Maiwald, A. Maestrini, J. J. Gill, S. C. Martin, D. Pukala, J. Ward, J. Kawamura, W. R. McGrath, W. Hatch, D. G. Harding, H. G. LeDuc, J. A. Stern, B. Bumble, L. A. Samoska, T. C. Gaier, R. Ferber, D. Miller, A. Karpov, J. Zmuidzinas, T. G. Phillips, N. R. Erickson, J. Swift, Y. Chung, R. Lai, and H. Wang, "THz frequency receiver instrumentation for Herschel's heterodyne instrument for far infrared (HIFI)," Proc. SPIE, IR Space Telescopes Instrum., vol. 4850, pp. 650-661, Mar. 2003.

[36] J. Horn, O. Siebertz, F. Schmulling, C. Kunz, R. Schieder, and G. Winnewisser, "A $4 \times 1 \mathrm{GHz}$ array acousto-optical spectrometer," Exp. Astron., vol. 9, no. 1, pp. 17-38, 1999.

[37] J. Hagen and D. Farley, "Digital-correlation techniques in radio science," Radio Sci., vol. 8, no. 8-9, pp. 775-784, 1973.

[38] W. Urry, D. Thornton, and J. Hudson, "The Hat Creek millimeter-wave hybrid spectrometer for interferometry," Publ. Astron. Soc. Pac., vol. 97, no. 594, pp. 745-751, 1985.

[39] E. Crete, M. Giard, L. Ravera, J. Noullet, P. d'Aguerre, M. Torres, and J. Mayvial, "Digital GaAs autocorrelator developed for space borne submillimeter astronomy," Exp. Astron., vol. 8, no. 3, pp. 239-255, 1998 .

[40] M. Torres, "A frequency-agile hybrid spectral corelator for mm-wave radio interferometry," Rev. Sci. Instrum., vol. 65, no. 5, pp. 1537-1540, 1994.

[41] S. Padin, T. Clark, M. Ewing, R. Finch, R. Lawrence, J. Navarro, S. Scott, N. Scoville, C. Seelinger, and T. Seling, "A high-speed digital correlator for radio astronomy," IEEE Trans. Instrum. Meas., vol. 42, pp. 793-798, Aug. 1993.

[42] D. M. Pozar, Microwave Engineering, 2nd ed. New York: Wiley, 1998.

[43] T. Phillips and D. Woody, "Millimeter-wave and submillimeter-wave receivers," Аnnu. Rev. Astron. Astrophys., vol. 20, pp. 285-321, 1982.

[44] J. Archer, "Low-noise heterodyne receivers for near-millimeter wave radio astronomy," Proc. IEEE, vol. 73, pp. 109-130, Jan. 1985.

[45] R. Blundell and C.-Y. E. Tong, "Submillimeter receivers for radio astronomy," Proc. IEEE, vol. 80, pp. 1702-1720, Nov. 1992.

[46] T. Crowe, R. Mattauch, H. Roser, W. Bishop, W. Peatman, and X. Liu, "GaAs Schottky diodes for THz mixing applications," Proc. IEEE, vol. 80, pp. 1827-1841, Nov. 1992.

[47] J. Bock, C. Bradford, L. Earle, J. Glenn, B. Naylor, H. Nguyen, and J. Zmuidzinas, "The case for space-borne far-infrared line surveys," in New Concepts for Far-Infrared and Submillimeter Space Astronomy, D. Benford and D. Leisawitz, Eds. Washington, DC: NASA, 2003, vol. NASA/CP-2003-212 233, p. 205.

[48] C. M. Caves, "Quantum limits on noise in linear amplifiers," Phys. Rev. D, vol. 26, pp. 1817-1839, 1982.

[49] J. Zmuidzinas, "Thermal noise and correlations in photon detection," Appl. Opt., vol. 42, no. 25, pp. 4989-5008, Sept. 2003.

[50] - "Progress in coherent detection methods," in The Physics and Chemistry of the Interstellar Medium, V. Ossenkopf et al., Eds. Herdecke, Germany: GCA-Verlag, 1999, pp. 423-430.

[51] — "The role of coherent detection," in New Concepts for FarInfrared and Submillimeter Space Astronomy, D. Benford and D. Leisawitz, Eds. Washington, DC: NASA, 2003, vol. NASA/CP2003-212 233, p. 329.

[52] - "Coherent detection and SIS mixers," in Proc. Far-IR, Sub-MM, and MM Detector Workshop, vol. NASA/CP-2003-211408, J. Wolf, J. Farhoomand, and C. R. McCreight, Eds., 2003, pp. 149-154.

[53] S. Padin, S. L. Scott, D. P. Woody, N. Z. Scoville, T. V. Seling, R. P. Finch, C. J. Giovanine, and R. P. Lawrence, "The Owens Valley millimeter array," Publ. Astron. Soc. Pac., vol. 103, pp. 461-467, May 1991.

[54] S. Guilloteau, J. Delannoy, D. Downes, A. Greve, M. Guelin, R. Lucas, D. Morris, S. J. E. Radford, J. Wink, J. Cernicharo, T. Forveille, S. Garcia-Burillo, R. Neri, J. Blondel, A. Perrigourad, D. Plathner, and M. Torres, "The IRAM interferometer on Plateau de Bure,” Astron. Astrophys., vol. 262, pp. 624-633, Sept. 1992.
[55] W. J. Welch, D. D. Thornton, R. L. Plambeck, M. C. H. Wright, J. Lugten, L. Urry, M. Fleming, W. Hoffman, J. Hudson, W. T. Lum, J. R. Forster, N. Thatte, X. Zhang, S. Zivanovic, L. Snyder, R. Crutcher, K. Y. Lo, B. Wakker, M. Stupar, R. Sault, Y. Miao, R. Rao, K. Wan, H. R. Dickel, L. Blitz, S. N. Vogel, L. Mundy, W. Erickson, P. J. Teuben, J. Morgan, T. Helfer, L. Looney, E. de Gues, A. Grossman, J. E. Howe, M. Pound, and M. Regan, "The Berkeley-Illinois-Maryland-Association millimeter array," Pub. Astron. Soc. Pac., vol. 108, pp. 93-103, Jan. 1996.

[56] A. Wooten, "Atacama large millimeter array (ALMA)," Proc. SPIE, Large Ground-Based Telescopes, vol. 4837, pp. 110-118, Feb. 2003.

[57] F. N. Owen. (1982, Sept.) The concept of a millimeter array. [Online]. Available: http://www.alma.nrao.edu/memos

[58] J. R. Tucker and M. J. Feldman, "Quantum detection at millimeter wavelengths," Rev. Mod. Phys., vol. 57, no. 4, pp. 1055-1113, 1985.

[59] Historical information about the MMA, A. Wootten. [Online]. Available: http://www.cv.nrao.edu/ awootten/mmaimcal/mmahistory.html

[60] G. A. Blake, E. C. Sutton, C. R. Masson, and T. G. Phillips, "Molecular abundances in OMC-1-The chemical composition of interstellar molecular clouds and the influence of massive star formation," Astrophys. J., vol. 315, pp. 621-645, Apr. 1987.

[61] P. Schilke, D. J. Benford, T. R. Hunter, D. C. Lis, and T. G. Phillips, "A line survey of orion-KL from 607 to $725 \mathrm{GHz}$," Astrophys. J. (Suppl.), vol. 132, pp. 281-364, Feb. 2001.

[62] S. D. Doty, E. F. van Dishoeck, F. F. S. van der Tak, and A. M. S. Boonman, "Chemistry as a probe of the structures and evolution of massive star-forming regions," Astron. Astrophys., vol. 389, pp. 446-463, July 2002.

[63] J. Pardo, J. Cernicharo, and E. Serabyn, "Atmospheric transmission at microwaves (ATM): An improved model for millimeter/submillimeter applications," IEEE Trans. Antennas Propagat., vol. 49, pp. 1683-1694, Dec. 2001.

[64] J. Zmuidzinas, G. A. Blake, J. Carlstrom, J. Keene, D. Miller, P. Schilke, and N. G. Ugras, "Submillimeter spectroscopy of interstellar hydrides," in Proc. Airborne Astronomy Symp. Galactic Ecosystem: From Gas to Stars to Dust, vol. 73, M. R. Haas, J. A. Davidson, and E. F. Erickson, Eds., 1995, pp. 33-40.

[65] G. J. Melnick, "The submillimeter wave astronomy satellite: Instrument and mission description," Adv. Space Res., vol. 30, pp. 2051-2057, 2002.

[66] A. M. S. Boonman, S. D. Doty, E. F. van Dishoeck, E. A. Bergin, G. J. Melnick, C. M. Wright, and R. Stark, "Modeling gas-phase $\mathrm{H}_{2} \mathrm{O}$ between $5 \mu \mathrm{m}$ and $540 \mu \mathrm{m}$ toward massive protostars," Astron. Astrophys., vol. 406, pp. 937-955, Aug. 2003.

[67] C. Ceccarelli, A. C. A. Boogert, A. G. G. M. Tielens, E. Caux, M. R. Hogerheijde, and B. Parise, "Submm/FIR CO line emission from the disk of the Class I protostar EL 29," Astron. Astrophys., vol. 395, pp. 863-871, Dec. 2002.

[68] E. Dartois, A. Dutrey, and S. Guilloteau, "Structure of the DM Tau outer disk: Probing the vertical kinetic temperature gradient," Astron. Astrophys., vol. 399, pp. 773-787, Feb. 2003.

[69] G. J. Melnick, D. A. Neufeld, K. E. S. Ford, D. J. Hollenbach, and M. L. N. Ashby, "Discovery of water vapor around IRC + 10216 as evidence for comets orbiting another star," Nature, vol. 412, pp. 160-163, July 2001.

[70] D. B. Sanders and I. F. Mirabel, "Luminous infrared galaxies," Аnпи. Rev. Astron. Astrophys., vol. 34, pp. 749-792, 1996.

[71] M. K. Crawford, R. Genzel, C. H. Townes, and D. M. Watson, "Farinfrared spectroscopy of galaxies-The 158 micron $\mathrm{C}^{+}$line and the energy balance of molecular clouds," Astrophys. J., vol. 291, pp. 755-771, Apr. 1985.

[72] A. I. Harris, J. Stutzki, U. U. Graf, A. P. G. Russell, R. Genzel, and R. E. Hills, "First observations of the CO $J=6-5$ transition in starburst galaxies," Astrophys. J. (Lett.), vol. 382, pp. L75-L79, Dec. 1991.

[73] J. Fischer, M. L. Luhman, S. Satyapal, M. A. Greenhouse, G. J. Stacey, C. M. Bradford, S. D. Lord, J. R. Brauher, S. J. Unger, P. E. Clegg, H. A. Smith, G. Melnick, J. W. Colbert, M. A. Malkan, L. Spinoglio, P. Cox, V. Harvey, J.-P. Suter, and V. Strelnitski, "ISO Far-IR spectroscopy of IR-bright galaxies and ULIRGs," Astrophys. Space Sci., vol. 266, pp. 91-98, 1999.

[74] R. Q. Mao, C. Henkel, A. Schulz, M. Zielinsky, R. Mauersberger, H. Störzer, T. L. Wilson, and P. Gensheimer, "Dense gas in nearby galaxies. XIII. CO submillimeter line emission from the starburst galaxy M 82," Astron. Astrophys., vol. 358, pp. 433-450, June 2000. 
[75] J. S. Ward, J. Zmuidzinas, A. I. Harris, and K. G. Isaak, " $\mathrm{A}^{12} \mathrm{CO}$ $J=6-5$ Map of M82: The significance of warm molecular gas," Astrophys. J., vol. 587, pp. 171-185, Apr. 2003.

[76] C. M. Bradford, T. Nikola, G. J. Stacey, A. D. Bolatto, J. M. Jackson, M. L. Savage, J. A. Davidson, and S. J. Higdon, " $\mathrm{CO}(J=7 \rightarrow 6)$ observations of NGC 253: Cosmic-ray-heated warm molecular gas," Astrophys. J., vol. 586, pp. 891-901, Apr. 2003.

[77] N. Scoville, "The central regions of galaxies," in Proc. Science with Atacama Large Millimeter Array Conf., vol. 235, A. Wooten, Ed., 2001, p. 281.

[78] P. M. Solomon, D. Downes, and S. J. E. Radford, "Warm molecular gas in the primeval galaxy IRAS $10214+4724$," Astrophys. J. (Lett.), vol. 398, pp. L29-L32, Oct. 1992.

[79] D. Downes and P. M. Solomon, "Molecular gas and dust at $z=2.6$ in SMM J14011+0252: A strongly lensed ultraluminous galaxy, not a huge massive disk," Astrophys. J., vol. 582, pp. 37-48, Jan. 2003.

[80] R. Genzel, A. J. Baker, L. J. Tacconi, D. Lutz, P. Cox, S. Guilloteau, and A. Omont, "Spatially resolved millimeter interferometry of SMM J02399-0136: A very massive galaxy at $z=2.8$," Astrophys. J., vol. 584, pp. 633-642, Feb. 2003.

[81] K. Irwin, "An application of electrothermal feedback for high-resolution cryogenic particle-detection," Appl. Phys. Lett., vol. 66, no. 15, pp. 1998-2000, 1995.

[82] S. Lee, J. Gildemeister, W. Holmes, A. Lee, and P. Richards, "Voltage-biased superconducting transition-edge bolometer with strong electrothermal feedback operated at $370 \mathrm{mK}$," Appl. Opt., vol. 37 , no. 16 , pp. 3391-3397, 1998.

[83] K. Irwin, G. Hilton, D. Wollman, and J. Martinis, "X-ray detection using a superconducting transition-edge sensor microcalorimeter with electrothermal feedback," Appl. Phys. Lett., vol. 69, no. 13, pp. 1945-1947, 1996

[84] B. Cabrera, R. Clarke, P. Colling, A. Miller, S. Nam, and R. Romani, "Detection of single infrared, optical, and ultraviolet photons using superconducting transition edge sensors," Appl. Phys. Lett., vol. 73, no. 6, pp. 735-737, 1998.

[85] A. Miller, B. Cabrera, R. Romani, E. Figueroa-Feliciano, S. Nam, and R. Clarke, "Development of wide-band, time and energy resolving, optical photon detectors with application to imaging astronomy," Nucl. Instrum. Meth., vol. A444, no. 1-2, pp. 445-448, 2000.

[86] K. Irwin, G. Hilton, J. Martinis, S. Deiker, N. Bergren, S. Nam, D. Rudman, and D. Wollman, "A Mo-Cu superconducting transitionedge microcalorimeter with $4.5 \mathrm{eV}$ energy resolution at $6 \mathrm{keV}, " \mathrm{Nucl}$. Instrum. Meth., vol. A444, no. 1-2, pp. 184-187, 2000.

[87] D. A. Wollman, G. C. Hilton, K. D. Irwin, N. F. Bergren, D. A Rudman, D. E. Newbury, and J. M. Martinis, "Cryogenic microcalorimeters for X-ray microanalysis," in Proc. 1999 NCSL Workshop and Symp. (Nat. Conf. Standards Laboratories), 1999, pp. 811-819.

[88] D. Newbury, D. Wollman, G. Hilton, K. Irwin, N. Bergren, D. Rudman, and J. Martinis, "The approaching revolution in X-ray microanalysis: The microcalorimeter energy dispersive spectrometer," J. Radioanal. Nucl. Chem., vol. 244, no. 3, pp. 627-635, 2000.

[89] N. White and H. Tananbaum, "The constellation X-ray mission," Astrophys. Lett. Comm., vol. 39, no. 1-6, pp. 933-936, 1999.

[90] P. de Korte, "Cryogenic imaging spectrometers for X-ray astronomy," Nucl. Inst. Meth. A, vol. 444, no. 1-2, pp. 163-169, 2000.

[91] J. Gildemeister, A. Lee, and P. Richards, "Monolithic arrays of absorber-coupled voltage- biased superconducting bolometers," Appl. Phys. Lett., vol. 77, no. 24, pp. 4040-4042, 2000.

[92] D. J. Benford, G. M. Voellmer, J. A. Chervenak, K. D. Irwin, S. H. Moseley, R. A. Shafer, G. J. Stacey, and J. G. Staguhn, "Thousand-element multiplexed superconducting bolometer arrays," in Proc. Far-IR, Sub-MM, and MM Detector Workshop, vol. NASA/CP-2003-211 408, J. Wolf, J. Farhoomand, and C. R. McCreight, Eds., 2003, pp. 272-275.

[93] W. Duncan, W. S. Holland, M. D. Audley, M. Cliffe, T. Hodson, B. D. Kelly, X. Gao, D. C. Gostick, M. MacIntosh, H. McGregor, T. Peacocke, K. D. Irwin, G. C. Hilton, S. W. Deiker, J. Beier, C. D. Reintsema, A. J. Walton, W. Parkes, T. Stevenson, A. M. Gundlach, C. Dunare, and P. A. R. Ade, "SCUBA-2: Developing the detectors," in Proc. SPIE, Millimeter and Submillimeter Detectors for Astronomy, vol. 4855, T. G. Phillips and J. Zmuidzinas, Eds., Feb. 2003, pp. 19-29.

[94] J. Gildemeister, A. Lee, and P. Richards, "A fully lithographed voltage-biased superconducting spiderweb bolometer," Appl. Phys. Lett., vol. 74, no. 6, pp. 868-870, 1999.
[95] S. Schwarz and B. Ulrich, "Antenna-coupled IR detectors," J. Appl. Phys., vol. 48, no. 5, pp. 1870-1873, 1977.

[96] M. Nahum and P. Richards, "Design analysis of a novel low-temperature bolometer," IEEE Trans. Magn., vol. 27, pp. 2484-2487, Mar. 1991.

[97] J. Mees, M. Nahum, and P. Richards, "New designs for antennacoupled superconducting bolometers," Appl. Phys. Lett., vol. 59, no. 18, pp. 2329-2331, 1991

[98] J. J. Bock, A. Goldin, C. Hunt, A. E. Lange, H. G. LeDuc, P. K. Day, A. Vayonakis, and J. Zmuidzinas, "Integrated focal plane arrays for millimeter-wave astronomy," in Proc. 9th Int. Workshop Low Temperature Detectors, vol. 605, F. S. Porter, D. McCammon, M. Galeazzi, and C. K. Stahle, Eds., 2002, pp. 243-246.

[99] J. J. Bock, P. Day, A. Goldin, H. G. LeDuc, C. Hunt, A. Lange, A. Vayonakis, and J. Zmuidzinas, "Antenna-coupled bolometer arrays for astrophysics," in Proc. Far-IR, Sub-MM, and MM Detector Workshop, vol. NASA/CP-2003-211 408, J. Wolf, J. Farhoomand, and C. R. McCreight, Eds., 2003, pp. 224-229.

[100] C. Hunt, A. Lange, A. Vayonakis, J. Zmuidzinas, J. J. Bock, P. K. Day, A. Goldin, and H. G. LeDuc, "Transition-edge superconducting antenna-coupled bolometer," in Proc. Far-IR, Sub-MM, and MM Detector Workshop, vol. NASA/CP-2003-211 408, J. Wolf, J. Farhoomand, and C. R. McCreight, Eds., 2003, pp. 281-283.

[101] C. L. Hunt, J. J. Bock, P. K. Day, A. Goldin, A. E. Lange, H. G. LeDuc, A. Vayonakis, and J. Zmuidzinas, "Transition-edge superconducting antenna-coupled bolometer," in Proc. SPIE, Millimeter and Submillimeter Detectors for Astronomy, vol. 4855, T. G. Phillips and J. Zmuidzinas, Eds., Feb. 2003, pp. 318-321.

[102] A. T. Lee, S. Cho, J. M. Gildemeister, N. Halverson, W. L. Holzapfel, J. Mehl, M. J. Myers, T. Lanting, P. L. Richards, E. Rittweger, D. Schwan, H. Spieler, and H. Tran, "Voltage-biased TES bolometers for the far-infrared to millimeter wavelength range," in Proc. SPIE, Millimeter and Submillimeter Detectors for Astronomy, vol. 4855, T. G. Phillips and J. Zmuidzinas, Eds., Feb. 2003, pp. 129-135.

[103] G. Chattopadhyay and J. Zmuidzinas, "A dual-polarized slot antenna for millimeter waves," IEEE Trans. Antennas Propagat., vol. 46, pp. 737-737, May 1998.

[104] M. J. Myers, A. T. Lee, P. L. Richards, D. Schwan, J. T. Skidmore, A. D. Smith, H. Spieler, and J. Yoon, "Antenna-coupled arrays of voltage-biased superconducting bolometers," in Proc. 9th Int. Workshop Low Temperature Detectors, vol. 605, F. S. Porter, D. McCammon, M. Galeazzi, and C. K. Stahle, Eds., 2002, pp. 247-250.

[105] A. Goldin, J. J. Bock, C. Hunt, A. E. Lange, H. G. LeDuc, P. K. Day, A. Vayonakis, and J. Zmuidzinas, "SAMBA: Superconducting antenna-coupled multi-frequency bolometric array," in Proc. 9th Int. Workshop Low Temperature Detectors, vol. 605, F. S. Porter, D. McCammon, M. Galeazzi, and C. K. Stahle, Eds., 2002, pp. 251-254.

[106] A. Goldin, J. J. Bock, C. Hunt, A. Lange, H. G. LeDuc, A. Vayonakis, and J. Zmuidzinas, "SAMBA: Superconducting antenna-coupled multi-frequency bolometric array," in Proc. Far-IR, Sub-MM, and MM Detector Workshop, vol. NASA/CP-2003-211 408, J. Wolf, J. Farhoomand, and C. R. McCreight, Eds., 2003, pp. 259-263.

[107] A. Goldin, J. J. Bock, C. L. Hunt, A. E. Lange, H. G. LeDuc, A. Vayonakis, and J. Zmuidzinas, "Design of broadband filters and antennas for SAMBA," in Proc. SPIE, Millimeter and Submillimeter Detectors for Astronomy, vol. 4855, T. G. Phillips and J. Zmuidzinas, Eds., Feb. 2003, pp. 163-171.

[108] A. Lee, P. Richards, S. Nam, B. Cabrera, and K. Irwin, "A superconducting bolometer with strong electrothermal feedback," Appl. Phys. Lett., vol. 69, no. 12, pp. 1801-1803, 1996.

[109] B. Karasik, W. McGrath, H. LeDuc, and M. Gershenson, "A hotelectron direct detector for radioastronomy," Supercond. Sci. Tech., vol. 12, no. 11, pp. 745-747, 1999.

[110] B. Karasik, W. McGrath, M. Gershenson, and A. Sergeev, "Photonnoise-limited direct detector based on disorder-controlled electron heating," J. Appl. Phys., vol. 87, no. 10, pp. 7586-7588, 2000

[111] B. S. Karasik, B. Dalaet, W. R. McGrath, J. Weu, M. Gershenson, and A. V. Sergeev, "Experimental study of superconducting hot-electron sensors for submm astronomy," IEEE Trans. Appl. Superconduct., vol. 13, pp. 188-191, June 2003.

[112] W. S. Holland, W. Duncan, B. D. Kelly, K. D. Irwin, A. J. Walton, P. A. R. Ade, and E. I. Robson, "SCUBA-2: A new generation submillimeter imager for the James Clerk Maxwell Telescope," in Proc. SPIE, Millimeter and Submillimeter Detectors for Astronomy, vol. 4855, T. G. Phillips and J. Zmuidzinas, Eds., Feb. 2003, pp. 1-18. 
[113] J. Chervenak, K. Irwin, E. Grossman, J. Martinis, C. Reintsema, and M. Huber, "Superconducting multiplexer for arrays of transition edge sensors," Appl. Phys. Lett., vol. 74, no. 26, pp. 4043-4045, 1999.

[114] P. de Korte, J. Beyer, S. Deiker, G. Hilton, K. Irwin, M. MacIntosh, S. Nam, C. Reintsema, L. Vale, and M. Huber, "Time-division superconducting quantum interference device multiplexer for transition-edge sensors," Rev. Sci. Instrum., vol. 74, no. 8, pp. 3807-3815, 2003.

[115] C. D. Reintsema, J. Beyer, S. W. Nam, S. Deiker, G. C. Hilton, K. Irwin, J. Martinis, J. Ullom, and L. R. Vale, "Prototype system for superconducting quantum interference device multiplexing of largeformat transition-edge sensor arrays," Rev. Sci. Instrum., vol. 74, no. 10, pp. 4500-4508, Oct. 2003.

[116] J. Yoon, J. Clarke, J. Gildemeister, A. Lee, M. Myers, P. Richards, and J. Skidmore, "Single superconducting quantum interference device multiplexer for arrays of low-temperature sensors," Appl. Phys. Lett., vol. 78, no. 3, pp. 371-373, 2001.

[117] M. Cunningham, J. Ullom, T. Miyazaki, S. Labov, J. Clarke, T. Lanting, A. Lee, P. Richards, J. Yoon, and H. Spieler, "High-resolution operation of frequency-multiplexed transition-edge photon sensors," Appl. Phys. Lett., vol. 81, no. 1, pp. 159-161, 2002.

[118] T. Lanting, H. Cho, J. Clarke, M. Dobbs, A. Lee, P. Richards, A. Smith, and H. Spieler, "A frequency-domain SQUID multiplexer for arrays of transition-edge superconducting sensors," IEEE Trans. Appl. Superconduct., vol. 13, pp. 626-629, June 2003.

[119] M. Kiviranta, J. Penttila, L. Gronberg, H. Seppa, and L. Suni, "DC and un SQUID's for readout of ac-biased transition-edge sensors," IEEE Trans. Appl. Superconduct., vol. 13, pp. 614-617, June 2003.

[120] J. van der Kuur, P. de Korte, H. Hoevers, W. Tiest, N. Baars, M. Ridder, E. Krouwer, M. Bruijn, M. Kiviranta, and H. Seppa, "AC biased TES-based X-ray microcalorimeter with an energy resolution of $6.3 \mathrm{eV}$ at $5.89 \mathrm{keV}$," IEEE Trans. Appl. Superconduct., vol. 13, pp. 638-642, June 2003.

[121] T. Miyazaki, M. Yamazaki, K. Futamoto, K. Mitsuda, R. Fujimoto, N. Iyomoto, T. Oshima, D. Audley, Y. Ishisaki, T. Kagei, T. Ohashi, N. Yamasaki, S. Shoji, H. Kudo, and Y. Yokoyama, "AC calorimeter bridge: A new multi-pixel readout method for TES calorimeter arrays," in Proc. 9th Int. Workshop Low Temperature Detectors, vol. 605, F. S. Porter, D. McCammon, M. Galeazzi, and C. K. Stahle, Eds., 2002, pp. 313-316.

[122] M. Tinkham, Introduction to Superconductivity, 2nd ed. New York: McGraw-Hill, 1996.

[123] E. Burstein, D. N. Langenberg, and B. N. Taylor, "Superconductors as quantum detectors for microwave and sub-millimeter-wave radiation," Phys. Rev. Lett., vol. 6, no. 3, pp. 92-94, Feb. 1961.

[124] C. Wilson, L. Frunzio, and D. Prober, "Time-resolved measurements of thermodynamic fluctuations of the particle number in a nondegenerate Fermi gas," Phys. Rev. Lett., vol. 87, no. 6, 2001. Art. no. 067004.

[125] G. Wood and B. White, "Pulses induced in tunneling currents between superconductors by alpha-particle bombardment," Appl. Phys. Lett., vol. 15, no. 8, pp. 237-239, 1969.

[126] — - "Detection of alpha-particles with superconducting tunnel junctions," Can. J. Phys., vol. 51, no. 19, pp. 2032-2046, 1973.

[127] M. Kurakado and H. Mazaki, "Quasiparticle excitation in a superconducting tunnel junction by alpha-particles," Phys. Rev. B, vol. 22, no. 1 , pp. $168-173,1980$.

[128] D. Twerenbold, "Giaever-type superconducting tunneling junctions as high-resolution X-ray-detectors," Europhys. Lett., vol. 1, no. 5, pp. 209-214, 1986.

[129] H. Kraus, T. Peterreins, F. Probst, F. von Feilitzsch, R. Mossbauer, V. Zacek, and E. Umlauf, "High-resolution X-ray-detection with superconducting tunnel-junctions," Europhys. Lett., vol. 1, no. 4, pp. 161-166, 1986.

[130] A. Peacock, P. Verhoeve, N. Rando, A. van Dordrecht, B. Taylor, C. Erd, M. Perryman, R. Venn, J. Howlett, D. Goldie, J. Lumley, and M. Wallis, "Single optical photon detection with a superconducting tunnel junction," Nature, vol. 381, no. 6578, pp. 135-137, 1996.

[131] F. Paresce, "Astronomy-Toward the ideal detector," Nature News Views, vol. 381, no. 6578, pp. 115-115, 1996.

[132] H. Kraus, "Superconductive bolometers and calorimeters," Supercond. Sci. Tech., vol. 9, no. 10, pp. 827-842, 1996

[133] J. Mather, "Astronomy-Super photon counters," Nature News Views, vol. 401, no. 6754, pp. 654-655, 1999.
[134] G. Angloher, P. Hettl, M. Huber, J. Jochum, F. von Feilitzsch, and R. Mossbauer, "Energy resolution of $12 \mathrm{eV}$ at $5.9 \mathrm{keV}$ from Al-superconducting tunnel junction detectors," J. Appl. Phys., vol. 89, no. 2, pp. 1425-1429, 2001.

[135] L. Li, L. Frunzio, C. Wilson, D. Prober, A. Szymkowiak, and S. Moseley, "Improved energy resolution of X-ray single photon imaging spectrometers using superconducting tunnel junctions," $J$. Appl. Phys., vol. 90, no. 7, pp. 3645-3647, 2001.

[136] P. Verhoeve, N. Rando, A. Peacock, D. Martin, and R. den Hartog, "Superconducting tunnel junctions as photoncounting imaging spectrometers from the optical to the x-ray band," Opt. Eng., vol. 41, no. 6, pp. 1170-1184, 2002.

[137] R. Schoelkopf, S. Moseley, C. Stahle, P. Wahlgren, and P. Delsing, "A concept for a submillimeter-wave single-photon counter," IEEE Trans. Appl. Superconduct., vol. 9, pp. 2935-2939, June 1999.

[138] T. Stevenson, F. Pellerano, C. Stahle, K. Aidala, and R. Schoelkopf, "Multiplexing of radio-frequency single-electron transistors," Appl. Phys. Lett., vol. 80, no. 16, pp. 3012-3014, 2002.

[139] R. Schoelkopf, P. Wahlgren, A. Kozhevnikov, P. Delsing, and D. Prober, "The radio-frequency single-electron transistor (RF-SET): A fast and ultrasensitive electrometer," Science, vol. 280, no. 5367, pp. 1238-1242, 1998.

[140] N. Bluzer, "Analysis of quantum superconducting kinetic inductance photodetectors," J. Appl. Phys., vol. 78, no. 12, pp. 7340-7351, 1995.

[141] A. Sergeev and M. Reizer, "Photoresponse mechanisms of thin superconducting films and superconducting detectors," Int. J. Mod. Phys. B, vol. 10, no. 6, pp. 635-667, 1996.

[142] B. A. Mazin, P. K. Day, J. Zmuidzinas, and H. G. LeDuc, "Multiplexable kinetic inductance detectors," in Proc. 9th Int. Workshop Low Temperature Detectors, vol. 605, F. S. Porter, D. McCammon, M. Galeazzi, and C. K. Stahle, Eds., 2002, pp. 309-312.

[143] J. Zmuidzinas, B. Mazin, A. Vayonakis, P. Day, and $H$. G. LeDuc, "Multiplexable kinetic inductance detectors," in Proc. Far-IR, Sub-MM, and MM Detector Workshop, vol. NASA/CP-2003-211408, J. Wolf, J. Farhoomand, and C. R. McCreight, Eds., 2003, pp. 326-329.

[144] R. N. Simons, Coplanar Waveguide Circuits, Components, and Systems. New York: Wiley-IEEE Press, 2001.

[145] P. Day, H. LeDuc, B. Mazin, A. Vayonakis, and J. Zmuidzinas, "A broadband superconducting detector suitable for use in large arrays," Nature, vol. 425, no. 6960, pp. 817-821, 2003.

[146] D. Prober, "Applied physics-To catch a photon," Nature, vol. 425, no. 6960, pp. 777-778, 2003.

[147] A. Gulian and D. Van Vechten, "Nonequilibrium dynamic conductivity of superconductors-An exploitable basis for high energy resolution x-ray detectors," Appl. Phys. Lett., vol. 67, no. 17, pp. 2560-2562, 1995.

[148] D. VanVechten, F. Porter, K. Wood, and A. Gulian, "Alternative nonequilibrium superconducting X-ray detectors," Nucl. Instrum. Meth., vol. A370, no. 1, pp. 34-37, 1996.

[149] J. Sauvageau and D. McDonald, "Superconducting kinetic inductance bolometer," IEEE Trans. Magn., vol. 25, pp. 1331-1334, Mar. 1989.

[150] J. Sauvageau, D. Mcdonald, and E. Grossman, "Superconducting kinetic inductance radiometer," IEEE Trans. Magn., vol. 27, pp. 2757-2760, Mar. 1991.

[151] E. Grossman, D. McDonald, and J. Sauvageau, "Far-infrared kineticinductance detectors," IEEE Trans. Magn., vol. 27, pp. 2677-2680, Mar. 1991.

[152] D. Osterman, R. Patt, D. Audley, and R. Kelley, "An X-ray microcalorimeter with kinetic inductance thermometer and dc SQUID read-out," J. Low Temp. Phys., vol. 93, no. 3-4, pp. 251-256, 1993.

[153] M. Audley, R. Kelley, and G. Rawley, "A prototype kinetic inductance thermometer for X-ray calorimetry," J. Low Temp. Phys., vol. 93, no. 3-4, pp. 245-250, 1993.

[154] H. Seppa, "Superconducting transition-edge bolometer in a resistive and in an inductive mode," IEEE Trans. Appl. Superconduct., vol. 11, pp. 759-761, Mar. 2001.

[155] A. Sergeev, V. Mitin, and B. Karasik, "Ultrasensitive hot-electron kinetic-inductance detectors operating well below the superconducting transition," Appl. Phys. Lett., vol. 80, no. 5, pp. 817-819, 2002.

[156] H. Matsuo, M. Takeda, T. Noguchi, S. Ariyoshi, and H. Akahori, "Development of a submillimeter-wave camera for the Atacama submillimeter telescope experiment," Proc. SPIE, Radio Telescopes, vol. 4015, pp. 228-236, July 2000.

[157] T. Van Duzer and C. W. Turner, Principles of Superconductive Devices and Circuits, 2nd ed. Upper Saddle River, NJ: Pearson, 1998. 
[158] M. Nahum and J. Martinis, "Ultrasensitive-hot-electron microbolometer," Appl. Phys. Lett., vol. 63, no. 22, pp. 3075-3077, 1993.

[159] L. Kuzmin, "Superconducting cold-electron bolometer with proximity traps," Microelectron. Eng., vol. 69, no. 2-4, pp. 309-316, 2003.

[160] D. Golubev and L. Kuzmin, "Nonequilibrium theory of a hot-electron bolometer with normal metal-insulator-superconductor tunnel junction," J. Appl. Phys., vol. 89, no. 11, pp. 6464-6472, 2001.

[161] D. Anghel and L. Kuzmin, "Capacitively coupled hot-electron nanobolometer as far-infrared photon counter," Appl. Phys. Lett., vol. 82, no. 2, pp. 293-295, 2003.

[162] D. Sandgren, D. Chouvaev, M. Tarasov, and L. Kuzmin, "Fabrication and optical characterization of the normal metal hot-electron microbolometer with Andreev mirrors," Physica C, vol. 372, pp. 444-447, 2002.

[163] M. Tarasov, M. Fominskii, A. Kalabukhov, and L. Kuzmin, "Experimental study of a normal-metal hot electron bolometer with capacitive coupling," JETP Lett., vol. 76, no. 8, pp. 507-510, 2002.

[164] M. Tarasov, S. Gudoshnikov, A. Kalabukhov, H. Seppa, M. Kiviranta, and L. Kuzmin, "Toward a dc SQUID read-out for the normal metal hot-electron microbolometer," Physica C, vol. 368, no. 1-4, pp. 161-165, 2002.

[165] A. H. Dayem and R. J. Martin, "Quantum interaction of microwave radiation with tunneling between superconductors," Phys. Rev. Lett., vol. 8, no. 6, pp. 246-248, Mar. 1962.

[166] P. K. Tien and J. P. Gordon, "Multiphoton process observed in the interaction of microwave fields with the tunneling between superconductor films," Phys. Rev., vol. 129, no. 3, pp. 647-651, Jan. 1963.

[167] P. L. Richards, T. M. Shen, R. E. Harris, and F. L. Lloyd, "Quasiparticle heterodyne mixing in SIS tunnel junctions," Appl. Phys. Lett., vol. 34, no. 5, pp. 345-347, Mar. 1979.

[168] G. J. Dolan, T. G. Phillips, and D. P. Woody, "Low-noise 115-GHz mixing in superconducting oxide-barrier tunnel junctions," Appl. Phys. Lett., vol. 34, no. 5, pp. 347-349, Mar. 1979.

[169] P. Richards and Q. Hu, "Superconducting components for infrared and millimeter-wave receivers," Proc. IEEE, vol. 77, pp. 1233-1246, Aug. 1989.

[170] M. J. Wengler, "Submillimeter-wave detection with superconducting tunnel diodes," Proc. IEEE, vol. 80, pp. 1810-1826, Nov. 1992.

[171] J. Carlstrom and J. Zmuidzinas, "Millimeter and submillimeter techniques," in Review of Radio Science 1993-1996, W. R. Stone, Ed. Oxford, U.K.: Oxford Univ. Press, 1996, pp. 839-882.

[172] J. Zmuidzinas, "Recent progress in submillimeter heterodyne receiver development," in Proc. Submillimeter and Far-Infrared Space Instrumentation, vol. ESA SP-388, 1996, pp. 151-154.

[173] M. J. Wengler and D. P. Woody, "Quantum noise in heterodyne detection,” IEEE J. Quantum Electron., vol. QE-23, pp. 613-622, May 1987.

[174] M. W. Johnson and M. J. Wengler, "Fully quantum analysis of radiation detection in an SIS," IEEE Trans. Appl. Superconduct., vol. 3, pp. 2242-2244, Mar. 1993.

[175] M. J. Wengler and D. P. Woody, "Experimental verification of the photodiode theory of SIS mixers," IEEE Trans. Appl. Superconduct., vol. 3, pp. 2230-2233, Nov. 1993.

[176] J. Tucker and M. Millea, "Photon detection in nonlinear tunneling devices," Appl. Phys. Lett., vol. 33, no. 7, pp. 611-613, 1978.

[177] J. Tucker, "Quantum limited detection in tunnel junction mixers," IEEE J. Quant. Electron., vol. 15, pp. 1234-1258, Nov. 1979.

[178] J. Tucker and M. Millea, "Superconductive tunneling devices as millimeter wave photon detectors," IEEE Trans. Magn., vol. 15, pp. 288-290, Jan. 1979.

[179] D. Rogovin and D. Scalapino, "Fluctuation phenomena in tunneljunctions," Ann. Phys., vol. 86, no. 1, pp. 1-90, 1974.

[180] C. A. Mears, Q. Hu, P. L. Richards, A. H. Worsham, D. E. Prober, and A. V. Räisänen, "Quantum-limited heterodyne detection of millimeter waves using superconducting tantalum tunnel junctions," Appl. Phys. Lett., vol. 57, no. 23, pp. 2487-2489, Mar. 1990.

[181] S. Withington and E. L. Kollberg, "Spectral-domain analysis of harmonic effects in superconducting quasiparticle mixers," IEEE Trans. Microwave Theory Tech., vol. 37, pp. 231-238, Jan. 1989.

[182] S. Withington and P. Kennedy, "Numerical procedure for simulating the large-signal quantum behavior of superconducting tunnel-junction circuits," IEE Proc. G, Circuits, Devices Syst., vol. 138, no. 1, pp. 70-76, 1991.
[183] L. R. D'Addario, "Noise parameters of SIS mixers," IEEE Trans. Microwave Theory Tech., vol. 36, pp. 1196-1206, July 1988.

[184] J. Ward, F. Rice, G. Chattopadhyay, and J. Zmuidzinas, "Supermix: A flexible software library for high-frequency circuit simulation, including SIS mixers and superconducting elements," in Proc. 10th Int. Symp. Space Terahertz Technology, 1999, pp. 268-281.

[185] F. Rice, J. Ward, J. Zmuidzinas, and G. Chattopadhyay, "Fast harmonic balance of SIS mixers with multiple junctions and superconducting circuits," in Proc. 10th Int. Symp. Space Terahertz Technology, 1999, pp. 282-297.

[186] L. R. D'Addario, "An SIS mixer for $90-120 \mathrm{GHz}$ with gain and wide bandwidth," Int. J. IR MM Waves, vol. 5, no. 11, pp. 1419-1442, 1984.

[187] R. Blundell, C. Tong, D. Papa, R. Leombruno, X. Zhang, S. Paine, J. Stern, H. LeDuc, and B. Bumble, "A wide-band fixed-tuned SIS receiver for 200-GHz operation," IEEE Trans. Microwave Theory Tech., vol. 43, pp. 933-937, Apr. 1995.

[188] C. Risacher, V. Vassilev, A. Pavolotsky, and V. Belitsky, "Waveguide-to-microstrip transition with integrated bias-T," IEEE Microwave Wireless Comp. Lett., vol. 13, pp. 262-264, July 2003.

[189] J. Kooi, G. Chattopadhyay, S. Withington, F. Rice, J. Zmuidzinas, C. Walker, and G. Yassin, "A full-height waveguide to thin-film microstrip transition with exceptional RF bandwidth and coupling efficiency," Int. J. IR MM Waves, vol. 24, no. 3, pp. 261-284, 2003.

[190] J. Kooi, J. Pety, B. Bumble, C. Walker, H. G. LeDuc, P. Schaeffer, and T. Phillips, "A 850-GHz waveguide receiver employing a niobium SIS junction fabricated on a $\mathrm{Si}_{3} \mathrm{~N}_{4}$ membrane," IEEE Trans. Microwave Theory Tech., vol. 46, pp. 151-161, Feb. 1998.

[191] M. Brandt, P. P. Muñoz, J. Stodolka, T. Tils, C. E. Honingh, and K. Jacobs, "Superconducting hot electron bolometers on fused quartz and on freestanding silicon nitride membrane strips," Proc. 6th Eur. Conf. Applied Superconductivity, 2003, to be published.

[192] R. B. Bass. (2004) Hot-electron bolometers on ultra-thin silicon chips with beam leads for a $585 \mathrm{GHz}$ receiver (Ph.D. dissertation) [Online]. Available: http://www.ece.virginia.edu/uvml/sis/ Papers/rbbpapers/eucas03SOI.pdf

[193] R. B. Bass, J. Z. Zhang, W. L. Bishop, A. W. Lichtenberger, and S.-K. Pan, "Beam lead quartz chips for superconducting millimeterwave circuits," in Proc. SPIE, Millimeter and Submillimeter Detectors for Astronomy, vol. 4855, T. G. Phillips and J. Zmuidzinas, Eds., Feb. 2003, pp. 415-426.

[194] R. Bass, J. Schultz, A. Lichtenberger, C. Walker, and J. Kooi. Beam lead fabrication using vacuum planarization. presented at Int. Symp. Space Terahertz Technology. [Online]. Available: http://soral.as. arizona.edu/STT03/index.html

[195] M. Wengler, D. Woody, R. Miller, and T. Phillips, "A low-noise receiver for millimeter and submillimeter wavelengths," Int. J. IR MM Waves, vol. 6, no. 8, pp. 697-706, 1985.

[196] R. Compton, R. McPhedran, Z. Popovic, G. Rebeiz, P. Tong, and D. Rutledge, "Bow-tie antennas on a dielectric half-space - theory and experiment," IEEE Trans. Antennas Propagat., vol. AP-35, pp. 622-631, June 1987.

[197] T. Büttgenbach, R. Miller, M. Wengler, D. Watson, and T. Phillips, "A broad-band low-noise SIS receiver for submillimeter astronomy," IEEE Trans. Microwave Theory Tech., vol. 36, pp. 1720-1726, Dec. 1988.

[198] A. Skalare, T. DeGraauw, and H. Van de Stadt, "A planar dipole array antenna with an elliptic lens," Microw. Opt. Tech. Lett., vol. 4 , no. 1, pp. 9-12, 1991.

[199] J. Zmuidzinas and H. G. LeDuc, "A $500 \mathrm{GHz}$ quasioptical slot antenna SIS mixer," in Superconducting Devices and their Applications, H. Koch and H. Lübbig, Eds. New York: Springer, 1991, pp. 395-398.

[200] - "Quasioptical slot antenna SIS mixers," IEEE Trans. Microwave Theory Tech., vol. 40, pp. 1797-1804, Sept. 1992.

[201] J. Zmuidzinas, N. G. Ugras, D. Miller, M. C. Gaidis, and H. G. LeDuc, "Low noise slot antenna SIS mixers," IEEE Trans. Appl. Superconduct., vol. 5, pp. 3053-3056, June 1995.

[202] G. Rebeiz, "Millimeter-wave and terahertz integrated-circuit antennas," Proc. IEEE, vol. 80, pp. 1748-1770, Nov. 1992.

[203] D. Filipovic, S. Gearhart, and G. Rebeiz, "Double-slot antennas on extended hemispherical and elliptic silicon dielectric lenses," IEEE Trans. Microwave Theory Tech., vol. 41, pp. 1738-1749, Oct. 1993.

[204] D. F. Filipovic, G. P. Gauthier, S. Raman, and G. M. Rebeiz, "Offaxis properties of silicon and quartz dielectric lens antennas," IEEE Trans. Antennas Propagat., vol. 45, pp. 760-766, May 1997. 
[205] M. van der Vorst, P. de Maagt, and M. Herben, "Effect of internal reflections on the radiation properties and input admittance of integrated lens antennas," IEEE Trans. Microwave Theory Tech., vol. 47, pp. 1696-1704, Sept. 1999.

[206] M. van der Vorst, P. de Maagt, A. Neto, A. Reynolds, R. Heeres, W. Luinge, and M. Herben, "Effect of internal reflections on the radiation properties and input impedance of integrated lens antennas: Comparison between theory and measurements," IEEE Trans. Microwave Theory Tech., vol. 49, pp. 1118-1125, June 2001.

[207] T. Vaupel, V. Hansen, and F. Schafer, "Radiation efficiency analysis of submillimeter-wave receivers based on a modified spectral domain integration technique," Radio Sci., vol. 38, no. 4, 2003. art. no.- 1074 .

[208] G. Chattopadhyay, F. Rice, D. Miller, H. G. LeDuc, and J. Zmuidzinas, "A 530-GHz balanced mixer," IEEE Microwave Guided Wave Lett., vol. 9, pp. 467-469, Nov. 1999.

[209] G. Chattopadhyay, D. Miller, H. LeDuc, and J. Zmuidzinas, "A dual-polarized quasioptical SIS mixer at $550 \mathrm{GHz}$," IEEE Trans. Microwave Theory Tech., vol. 48, pp. 1680-1686, Oct. 2000.

[210] A. Räisänen, W. McGrath, P. Richards, and F. L. Lloyd, "Broadband RF match to a millimeter-wave SIS quasiparticle mixer," IEEE Trans. Microwave Theory Tech., vol. 33, pp. 1495-1500, Dec. 1985.

[211] A. Kerr, S. Pan, and M. Feldman, "Integrated tuning elements for SIS mixers," Int. J. IR MM Waves, vol. 9, no. 2, pp. 203-212, 1988.

[212] T. Büttgenbach, H. LeDuc, P. Maker, and T. Phillips, "A fixed tuned broadband matching structure for submillimeter SIS receivers," IEEE Trans. Appl. Superconduct., vol. 2, pp. 165-175, Sept. 1992.

[213] V. Belitsky and M. Tarasov, "SIS junction reactance complete compensation,” IEEE Trans. Magn., vol. 27, pp. 2638-2641, Mar. 1991.

[214] J. Zmuidzinas, H. G. LeDuc, J. A. Stern, and S. R. Cypher, "Twojunction tuning circuits for submillimeter SIS mixers," IEEE Trans. Microwave Theory Tech., vol. 42, pp. 698-706, Apr. 1994.

[215] V. Belitsky, S. Jacobsson, L. Filippenko, and E. Kollberg, "Broad-band twin-junction tuning circuit for submillimeter SIS mixers," IEEE Microwave Opt. Tech. Lett., vol. 10, pp. 74-78, Oct. 1995.

[216] G. Yassin and S. Withington, "Electromagnetic models for superconducting millimeter-wave and sub-millimeter-wave microstrip transmission-lines," J. Phys. D, vol. 28, no. 9, pp. 1983-1991, 1995.

[217] V. Belitsky, C. Risacher, M. Pantaleev, and V. Vassilev. Superconducting microstrip line models at millimeter and submillimeter waves and their comparison. presented at 14th Int. Symp. Space Terahertz Technology. [Online]. Available: http://soral.as.arizona. edu/STT03/index.html

[218] A. Vayonakis, H. G. LeDuc, C. Luo, and J. Zmuidzinas, "Precision measurements of the millimeter-wave properties of superconducting thin-film microstrip lines," J. Appl. Phys., submitted for publication.

[219] A. R. Kerr, "Some fundamental and practical limits on broadband matching to capacitive devices, and the implications for SIS mixer design," IEEE Trans. Microwave Theory Tech., vol. 43, pp. 2-13, Jan. 1995.

[220] M. C. Gaidis, H. G. LeDuc, M. Bin, D. Miller, J. A. Stern, and J. Zmuidzinas, "Characterization of low-noise quasioptical SIS mixers for the submillimeter band," IEEE Trans. Microwave Theory Tech., vol. 44, pp. 1130-1139, July 1996.

[221] J. Kawamura, D. Miller, J. Chen, J. Zmuidzinas, B. Bumble, H. G. LeDuc, and J. A. Stern, "Very high current density Nb/AlN/Nb tunnel junctions for low-noise submillimeter mixers," Appl. Phys. Lett., vol. 76, no. 15, pp. 2119-2121, Apr. 2000.

[222] C. Tong, R. Blundell, B. Bumble, J. Stern, and H. LeDuc, "Quantum limited heterodyne-detection in superconducting nonlinear transmission-lines at submillimeter wavelengths," Appl. Phys. Lett., vol. 67, no. 9, pp. 1304-1306, 1995.

[223] C. Y. E. Tong, L. Chen, and R. Blundell, "Theory of distributed mixing and amplification in a superconducting quasiparticle nonlinear transmission line," IEEE Trans. Microwave Theory Tech., vol. 45, pp. 1086-1092, July 1997.

[224] M. Takeda and T. Noguchi, “A 200-285 GHz waveguide SIS mixer with an inhomogeneous distributed junction array," IEEE Trans. Microwave Theory Tech., vol. 50, pp. 2618-2623, Nov. 2002.

[225] G. Delange, J. Kuipers, and T. Klapwijk, "Superconducting resonator circuits at frequencies above the gap frequency," J. Appl. Phys., vol. 77, no. 4, pp. 1795-1804, 1995.

[226] B. Jackson and T. Klapwijk, "The current status of low-noise THz mixers based on SIS junctions," Physica $C$, vol. 372, no. 1, pp. 368-373, 2002.
[227] M. Bin, M. C. Gaidis, J. Zmuidzinas, T. G. Phillips, and H. G. LeDuc, "Low-noise $1 \mathrm{THz}$ niobium superconducting tunnel junction mixer with normal metal tuning circuit," Appl. Phys. Lett., vol. 68, pp. 1714-1716, 1996.

[228] — , "Quasioptical SIS mixers with normal metal tuning structures," IEEE Trans. Appl. Superconduct., vol. 7, pp. 3584-3588, June 1997.

[229] J. A. Stern, B. Bumble, H. G. LeDuc, J. W. Kooi, and J. Zmuidzinas, "Fabrication and DC characterization of NbTiN based SIS mixers for use between 600 and $1200 \mathrm{GHz}$," in Proc. 9th Int. Symp. Space Terahertz Technology, 1998, pp. 305-313.

[230] J. W. Kooi, J. A. Stern, G. Chattodpadhyay, H. G. LeDuc, B. Bumble, and J. Zmuidzinas, "Low-loss NbTiN films for THz SIS mixer tuning circuits," Int. J. IR and MM Waves, vol. 19, pp. 373-383, 1998.

[231] J. Zmuidzinas, J. W. Kooi, J. Kawamura, G. Chattodpadhyay, J. A. Stern, B. Bumble, and H. G. LeDuc, "Development of SIS mixers for 1 THz," Proc. SPIE, Adv. Technol. MMW, Radio, Terahertz Telescopes, vol. 3357, pp. 53-61, May 1998.

[232] J. Kawamura, J. Chen, D. Miller, J. Kooi, J. Zmuidzinas, B. Bumble, H. G. LeDuc, and J. A. Stern, "Low-noise submillimeter-wave NbTiN superconducting tunnel junction mixers," Appl. Phys. Lett., vol. 75, pp. 4013-4015, Dec. 1999.

[233] B. Bumble, H. G. LeDuc, J. A. Stern, and K. G. Megerian, "Fabrication of $\mathrm{Nb} / \mathrm{AlN}_{x} / \mathrm{NbTiN}$ junctions for SIS mixer applications," IEEE Trans. Appl. Superconduct., vol. 11, pp. 76-79, Mar. 2001.

[234] Y. Uzawa, Z. Wang, and A. Kawakami, "Terahertz NbN/AlN/NbN mixers with $\mathrm{Al} / \mathrm{SiO} / \mathrm{NbN}$ microstrip tuning circuits," Appl. Phys. Lett., vol. 73, no. 5, pp. 680-682, 1998.

[235] B. Jackson, A. Baryshev, G. de Lange, J. Gao, S. Shitov, N. Iosad, and T. Klapwijk, "Low-noise $1 \mathrm{THz}$ superconductor-insulator-superconductor mixer incorporating a $\mathrm{NbTiN} / \mathrm{SiO}_{2} / \mathrm{Al}$ tuning circuit," Appl. Phys. Lett., vol. 79, no. 3, pp. 436-438, 2001.

[236] Y. Uzawa, S. Miki, Z. Wang, A. Kawakami, M. Kroug, P. Yagoubov, and E. Kollberg, "Performance of a quasioptical NbN hot-electron bolometric mixer at terahertz frequencies," Supercond. Sci. Tech., vol. 15, no. 1, pp. 141-145, 2002.

[237] S. Shitov, B. Jackson, A. Baryshev, A. Markov, N. Iosad, J. Gao, and T. Klapwijk, "A low-noise double-dipole antenna SIS mixer at 1 THz," Physica C, vol. 372, no. 1, pp. 374-377, 2002.

[238] A. Karpov, D. Miller, F. Rice, J. Zmuidzinas, B. Bumble, J. A. Stern, and H. G. LeDuc, "Low noise $1.2 \mathrm{THz}$ SIS receiver," presented at the 8th Int. Superconductive Electronics Conf. (ISEC'01), Osaka, Japan.

[239] N. N. Iosad, M. Kroug, T. Zijlstra, A. B. Ermakov, B. D. Jackson, M. Zuiddam, F. E. Meijer, and T. M. Klapwijk, "Analysis of the fabrication process of $\mathrm{Nb} / \mathrm{Al}-\mathrm{AlN}{ }_{x} / \mathrm{Nb}$ tunnel junctions with low $R_{n} A$ values for SIS mixers," IEEE Trans. Appl. Superconduct., vol. 13, pp. 127-130, June 2003.

[240] W. Wild, J. M. Payne, V. Belitsky, T. W. Bradshaw, M. Carter, B. N. Ellison, M. Harman, J. Lamb, B. Lazareff, G. Moorey, A. H. Orlowska, S. Torchinsky, A. Baryshev, and R. Wade, "Receivers for ALMA: Preliminary design concepts," Proc. SPIE, Radio Telescopes, vol. 4015, pp. 320-327, July 2000.

[241] S. Padin, D. P. Woody, J. A. Stern, H. G. LeDuc, R. Blundell, C. E. Tong, and M. W. Pospieszalski, "An integrated SIS mixer and HEMT IF amplifier," IEEE Trans. Microwave Theory Tech., vol. 44, pp. 987-990, June 1996.

[242] A. Kerr, S.-K. Pan, A. Lichtenberger, N. Horner, J. E. Effland, and K. Crady, "A single-chip balanced SIS mixer for 200-300 GHz," in Proc. 11th Int. Symp. Space Terahertz Technology, 2000, pp. 251-259.

[243] V. Vassilev, V. Belitsky, and R. S. Booth, "New sideband separation SIS mixer for ALMA," Proc. SPIE, Radio Telescopes, vol. 4015, pp. 567-573, July 2000.

[244] E. F. Lauria, A. R. Kerr, M. W. Pospieszalski, S. Pan, J. E. Effland, and A. W. Lichtenberger, "A 200-300 GHz SIS mixer-preamplifier with $8 \mathrm{GHz}$ IF bandwidth," in 2001 IEEE Microwave Theory and Techniques Soc. Dig., pp. 1645-1648.

[245] — , (2001, June) A 200-300 GHz SIS mixer-preamplifier with 8 GHz IF bandwidth. [Online]. Available: http://www.alma.nrao.edu/ memos

[246] A. Baryshev, E. Lauria, R. Hesper, T. Zijlstra, and W. Wild. (2002, July) Fixed-tuned waveguide $0.6 \mathrm{THz}$ SIS mixer with wide band IF. [Online]. Available: http://www.alma.nrao.edu/memos 
[247] F. Rice, M. Sumner, J. Zmuidzinas, R. Hu, H. G. LeDuc, A. I. Harris, and D. Miller, "SIS mixer design for a broadband millimeter spectrometer suitable for rapid line surveys and redshift determinations," in Proc. SPIE, Millimeter and Submillimeter Detectors for Astronomy, vol. 4855, T. G. Phillips and J. Zmuidzinas, Eds., Feb. 2003, pp. 301-311.

[248] M. Salez, Y. Delorme, I. Peron, B. Lecomte, F. Dauplay, F. Boussaha, J. Spatazza, A. Feret, J. M. Krieg, and K. Schuster, "A 30\% bandwidth tunerless SIS mixer of quantum-limited sensitivity for Herschel/HIFI Band 1," in Proc. SPIE, Millimeter and Submillimeter Detectors for Astronomy, vol. 4855, T. G. Phillips and J. Zmuidzinas, Eds., Feb. 2003, pp. 402-414.

[249] S. Claude. Sideband-separating SIS mixer for ALMA band 7, 275-370 GHz. presented at Int. Symp. Space Terahertz Technology. [Online]. Available: http://soral.as.arizona.edu/STT03/index.html

[250] S. Asayama, K. Kimura, H. Iwashita, N. Sato, T. Takahashi, M. Saito, B. Ikenoue, H. Ishizaki, and N. Ukita, Preliminary tests of waveguide type sideband-separating SIS mixer for astronomical observation, Nov. 2003.

[251] C. C. Chin, D. Derdall, J. Sebesta, F. Jiang, P. Dindo, G. Rodrigues, D. Bond, S.-K. Pan, A. R. Kerr, E. Lauria, M. Posieszalski, J. Zhang, T. Cecil, and A. Lichtenberger, "A low noise $100 \mathrm{GHz}$ sideband-separating receiver," Int. J. IR MM Waves, vol. 25, no. 4, pp. 569-600, Apr. 2004 , to be published.

[252] ALMA memo series [Online]. Available: http://www.alma.nrao.edu/ memos

[253] Proc. 14th Int. Symp. Space Terahertz Technology, C. K. Walker, Ed., 2003, http://soral.as.arizona. edu/STT03/index.html.

[254] T. Phillips and K. Jefferts, "Low-temperature bolometer heterodyne receiver for millimeter wave astronomy," Rev. Sci. Instrum., vol. 44, no. 8, pp. 1009-1014, 1973.

[255] E. Gershenzon, G. Gol'tsman, Y. Gousev, A. Elantev, and A. Semenov, "Electromagnetic-radiation mixer based on electron heating in resistive state of superconductive $\mathrm{Nb}$ and $\mathrm{YBaCuO}$ films," IEEE Trans. Magn., vol. 27, pp. 1317-1320, Mar. 1991.

[256] E. Gerecht, C. Musante, Y. Zhuang, K. Yngvesson, T. Goyette, J. Dickinson, J. Waldman, P. Yagoubov, G. Gol'tsman, B. Voronov, and E. Gershenzon, "NbN hot electron bolometric mixers-a new technology for low-noise THz receivers," IEEE Trans. Microwave Theory Tech., vol. 47, pp. 2519-2527, Dec. 1999.

[257] J. Kawamura, T. Hunter, C. Tong, R. Blundell, D. Papa, F. Patt, W. Peters, T. Wilson, C. Henkel, G. Gol'tsman, and E. Gershenzon, "Ground-based terahertz CO spectroscopy toward Orion," Astron. Astrophys., vol. 394, no. 1, pp. 271-274, 2002.

[258] Y. Gousev, G. Gol'tsman, A. Semenov, E. Gershenzon, R. Nebosis, M. Heusinger, and K. Renk, "Broad-band ultrafast superconducting NbN detector for electromagnetic-radiation," J. Appl. Phys., vol. 75, no. 7, pp. 3695-3697, 1994.

[259] B. Karasik, G. Goltsman, B. Voronov, S. Svechnikov, E. Gershenzon, H. Ekstrom, S. Jacobsson, E. Kollberg, and K. Yngvesson, "Hot-electron quasioptical $\mathrm{NbN}$ superconducting mixer," IEEE Trans. Appl. Superconduct., vol. 5, pp. 2232-2235, June 1995.

[260] D. Prober, "Superconducting terahertz mixer using a transition-edge microbolometer," Appl. Phys. Lett., vol. 62, no. 17, pp. 2119-2121, 1993.

[261] D. W. Floet, J. Baselmans, T. Klapwijk, and J. Gao, "Resistive transition of niobium superconducting hot-electron bolometer mixers," Appl. Phys. Lett., vol. 73, no. 19, pp. 2826-2828, 1998.

[262] H. Merkel, P. Khosropanah, P. Yagoubov, and E. Kollberg, "A hotspot mixer model for phonon-cooled $\mathrm{NbN}$ hot electron bolometric mixers," IEEE Trans. Appl. Superconduct., vol. 9, pp. 4201-4204, June 1999.

[263] D. W. Floet, E. Miedema, J. J. A. Baselmans, T. M. Klapwijk, and J. R. Gao, "Resistive states of superconducting hot-electron bolometer mixers: Charge-imbalance vs. hotspot," IEEE Trans. Appl. Superconduct., vol. 9, pp. 3749-3752, June 1999.

[264] H. Merkel, P. Khosropanah, D. Floet, P. Yagoubov, and E. Kollberg, "Conversion gain and fluctuation noise of phonon-cooled hot-electron bolometers in hot-spot regime," IEEE Trans. Microwave Theory Tech., vol. 48, pp. 690-699, Apr. 2000.

[265] H. Merkel, P. Khosropanah, S. Cherednichenko, K. Yngvesson, A. Adam, and E. Kollberg, "A two-dimensional hot-spot mixer model for phonon-cooled hot electron bolometers," IEEE Trans. Appl. Superconduct., vol. 11, pp. 179-182, Mar. 2001.

[266] S. Cherednichenko, P. Khosropanah, E. Kollberg, M. Kroug, and H. Merkel, "Terahertz superconducting hot-electron bolometer mixers," Physica C, vol. 372, no. 1, pp. 407-415, 2002.
[267] A. Skalare, W. R. McGrath, P. M. Echternach, H. G. LeDuc, I. Siddiqi, A. Verevkin, and D. E. Prober, "Aluminum hot-electron bolometer mixers at submillimeter wavelengths," IEEE Trans. Appl. Superconduct., vol. 11, pp. 641-644, Mar. 2001.

[268] I. Siddiqi, A. Verevkin, D. E. Prober, A. Skalare, B. S. Karasik, W. R. McGrath, P. Echternach, and H. G. LeDuc, "Noise and conversion efficiency of aluminum superconducting hot-electron bolometer mixer," IEEE Trans. Appl. Superconduct., vol. 11, pp. 958-961, Mar. 2001.

[269] I. Siddiqi, A. Verevkin, D. Prober, A. Skalare, W. McGrath, P. Echternach, and H. LeDuc, "Heterodyne mixing in diffusion-cooled superconducting aluminum hot-electron bolometers," J. Appl. Phys., vol. 91, no. 7, pp. 4646-4654, 2002.

[270] A. Skalare, W. R. McGrath, B. Bumble, and H. G. LeDuc, "Speed measurements of diffusion-cooled tantalum bolometers," IEEE Trans. Appl. Superconduct., vol. 13, pp. 160-163, June 2003.

[271] C.-Y. E. Tong, D. Meledin, D. Loudkov, R. Blundell, N. Erickson, J. Kawamura, I. Mehdi, and G. Gol'tsman, "A 1.5 THz hot-electron bolometer mixer operated by a planar diode based local oscillator," in 2003 IEEE Microwave Theory and Techniques Soc. Dig., 2003, pp. 751-754.

[272] A. B. Kaul, B. Bumble, K. A. Lee, H. G. LeDuc, F. Rice, and J. Zmuidzinas, "Fabrication of wide-IF 200-300 GHz SIS mixers with suspended metal beam leads formed on SOI," J. Vac. Sci. Technol. $B, 2004$, to be published.

[273] S. Withington, J. Leech, G. Yassin, K. Isaak, B. Jackson, J. Gao, and T. Klapwijk, "A $350 \mathrm{GHz}$ radial-probe SIS mixer for astronomical imaging arrays," Int. J. IR MM Waves, vol. 22, no. 9, pp. 1305-1312, 2001.

[274] D. Rutledge and M. Muha, "Imaging antenna arrays," IEEE Trans. Antennas Propagat., vol. AP-30, pp. 535-540, July 1982.

[275] M. Gurvitch, M. A. Washington, and H. A. Huggins, "High quality refractory Josephson tunnel junctions utilizing thin aluminum layers," Appl. Phys. Lett., vol. 42, pp. 472-474, Mar. 1983.

[276] C. Honingh, S. Haas, D. Hottgenroth, K. Jacobs, and J. Stutzki, "Low noise broadband fixed tuned SIS waveguide mixers at 660 and 800 GHz," IEEE Trans. Appl. Superconduct., vol. 7, pp. 2582-2586, June 1997.

[277] B. Jackson, N. Iosad, G. de Lange, A. Baryshev, W. Laauwen, J. G. $\mathrm{JR}$, and T. Klapwijk, "NbTiN/SiO $/ 2 / \mathrm{Al}$ tuning circuits for low-noise 1 THz SIS mixers," IEEE Trans. Appl. Superconduct., vol. 11, pp. 653-656, Mar. 2001.

[278] A. Karpov, "Receivers for ground-based mm wave radio telescopes," Proc. SPIE, Adv. Technol. MMW, Radio, Terahertz Telescopes, vol. 3357, pp. 593-608, 1998.

[279] A. Karpov, J. Blondel, M. Voss, and K.-H. Gundlach, "A three photon noise SIS heterodyne receiver at submillimeter wavelength," IEEE Trans. Appl. Superconduct., vol. 9, pp. 4456-4459, June 1999.

[280] A. Karpov, private communication, 2003.

[281] J. Kooi, J. Kawamura, J. Chen, G. Chattopadhyay, J. Pardo, J. Zmuidzinas, T. Phillips, B. Bumble, J. Stern, and H. LeDuc, "A low noise NbTiN-based $850 \mathrm{GHz}$ SIS receiver for the Caltech submillimeter observatory," Int. J. IR MM Waves, vol. 21, no. 9, pp. 1357-1373, 2000.

[282] B. Lazareff, D. Billon-Pierron, A. Navarrini, and I. Peron, "Design and characterization of 225-370 GHz DSB and 247-360 GHz SSB full height waveguide SIS mixers," J. de Physique IV, vol. 12, no. PR3, pp. 161-164, 2002.

[283] C. Tong, R. Blundell, S. Paine, D. Papa, J. Kawamura, X. Zhang, J. Stern, and H. LeDuc, "Design and characterization of a 250-350-GHz fixed-tuned superconductor-insulator-superconductor receiver," IEEE Trans. Microwave Theory Tech., vol. 44, pp. 1548-1556, Sept. 1996.

[284] Y. Uzawa, A. Kawakami, S. Miki, and Z. Wang, "Performance of all-NbN quasioptical SIS mixers for the terahertz band," IEEE Trans. Appl. Superconduct., vol. 11, pp. 183-186, Mar. 2001.

[285] S. Cherednichenko, M. Kroug, H. Merkel, P. Khosropanah, A. Adam, E. Kollberg, D. Loudkov, G. Gol'tsman, B. Voronov, H. Richter, and H. Huebers, "1.6 THz heterodyne receiver for the far infrared space telescope," Physica C, vol. 372, no. 1, pp. 427-431, 2002.

[286] B. Karasik, M. Gaidis, W. McGrath, B. Bumble, and H. LeDuc, "Low noise in a diffusion-cooled hot-electron mixer at $2.5 \mathrm{THz}$," Appl. Phys. Lett., vol. 71, no. 11, pp. 1567-1569, 1997. 
[287] J. Kawamura, R. Blundell, C. Tong, G. Goltsman, E. Gershenzon, B. Voronov, and S. Cherednichenko, "Low noise NbN lattice-cooled superconducting hot-electron bolometric mixers at submillimeter wavelengths," Appl. Phys. Lett., vol. 70, no. 12, pp. 1619-1621, 1997.

[288] J. Kawamura, C. Tong, R. Blundell, D. Papa, T. Hunter, G. Gol'tsman, S. Cherednichenko, B. Voronov, and E. Gershenzon, "An $800 \mathrm{GHz} \mathrm{NbN}$ phonon-cooled hot-electron bolometer mixer receiver," IEEE Trans. Appl. Superconduct., vol. 9, pp. 3753-3756, June 1999.

[289] J. Kawamura, R. Blundell, C. Tong, D. Papa, T. Hunter, S. Paine, F. Patt, G. Gol'tsman, S. Cherednichenko, B. Voronov, and E. Gershenzon, "Superconductive hot-electron-bolometer mixer receiver for 800-GHz operation," IEEE Trans. Microwave Theory Tech., vol. 48, pp. 683-689, Apr. 2000.

[290] J. Kawamura, C. Tong, R. Blundell, D. Papa, T. Hunter, F. Patt, G. Gol'tsman, and E. Gershenzon, "Terahertz-frequency waveguide NbN hot-electron bolometer mixer," IEEE Trans. Appl. Superconduct., vol. 11, pp. 952-954, Mar. 2001.

[291] M. Kroug, S. Cherednichenko, H. Merkel, E. Kollberg, B. Voronov, G. Gol'tsman, H. Huebers, and H. Richter, "NbN hot electron bolometric mixers for terahertz receivers," IEEE Trans. Appl. Superconduct., vol. 11, pp. 962-965, Mar. 2001.

[292] A. Semenov, H. Hubers, H. Richter, M. Birk, M. Krocka, U. Mair, Y. Vachtomin, M. Finkel, S. Antipov, B. Voronov, K. Smirnov, N. Kaurova, V. Drakinski, and G. Gol'tsman, "Superconducting hotelectron bolometer mixer for terahertz heterodyne receivers," IEEE Trans. Appl. Superconduct., vol. 13, pp. 168-171, June 2003.

[293] A. Skalare, W. R. McGrath, B. Bumble, H. G. LeDuc, P. J. Burke, A. A. Verheijen, and D. E. Prober, "A heterodyne receiver at $533 \mathrm{GHz}$ using a diffusion-cooled superconducting hot electron bolometer mixer," IEEE Trans. Appl. Superconduct., vol. 5, pp. 2236-2239, June 1995.

[294] A. Skalare, W. McGrath, B. Bumble, and H. LeDuc, "Receiver measurements at $1267 \mathrm{GHz}$ using a diffusion-cooled superconducting transition-edge bolometer," IEEE Trans. Appl. Superconduct., vol. 7, pp. 3568-3571, June 1997.

[295] M. Hajenius, J. J. A. Baselmans, J. R. Gao, T. M. Klapwijk, P. A. J. de Korte, and G. Gol'tsman, "Low noise $\mathrm{NbN}$ superconducting hot electron bolometer mixers at 1.9 and $2.5 \mathrm{THz}$," Supercond. Sci. Tech., vol. 17, pp. S224-S228, Mar. 2004.

[296] P. Yagoubov, M. Kroug, H. Merkel, E. Kollberg, J. Schubert, and $\mathrm{H}$. Hubers, "NbN hot electron bolometric mixers at frequencies between 0.7 and 3.1 THz," Supercond. Sci. Tech., vol. 12, no. 11, pp. 989-991, 1999.

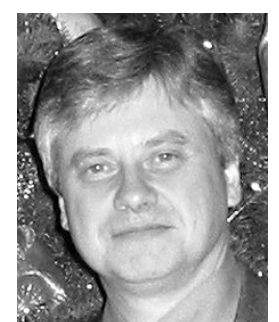

Jonas Zmuidzinas (Member, IEEE) received the B.S. degree in physics from the California Institute of Technology (Caltech), Pasadena, in 1981 and the $\mathrm{Ph} . \mathrm{D}$. degree in physics from the University of California, Berkeley, in 1987 under the direction of Dr. A. L. Betz and Prof. R. L. Genzel with Prof. P. L. Richards serving on his thesis committee.

He was a Postdoctoral Fellow in 1988-1989 at the University of Illinois, Urbana-Champaign, where he worked on the design and fabrication of submillimeter superconductor-insulator-superconductor (SIS) mixers with Profs. K.-Y. Lo and D. Van Harlingen. He joined the physics faculty at Caltech in 1989, where he currently serves as a Professor of Physics. His research efforts include the development of superconducting detectors and mixers, as well as the construction of submillimeter instruments for groundbased, airborne, and space telescopes.

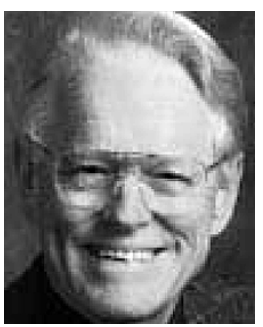

Paul L. Richards received the A.B. degree in physics from Harvard University, Cambridge, MA, in 1956 and the Ph.D. degree in solid state physics from the University of California, Berkeley, in 1960.

He was a Postdoctoral Researcher at Cambridge University, Cambridge, U.K., in 1959-1960, a Member of the Technical Staff at the Bell Telephone Laboratories from 1960 to 1966, and Professor of Physics at the University of California, Berkeley, from 1966 to the present. He has also been a Visiting Scientist at Cambridge University, the Max Planck Institutes for Solid State Physics at Stuttgart and Radio Astronomy at Bonn, the Ecole Normale Superieure in Paris, the Paris Observatory, and the University of Rome. With students and collaborators, he has published more than 400 papers on infrared and millimeter-wave physics, including the development of measurement techniques, especially new detectors, and the application of these techniques to many scientific problems.

Dr. Richards is a Member of the National Academy of Sciences and the American Academy of Arts and Science. He has received fellowships from the Alexander von Humboldt Foundation, the J. S. Guggenheim Memorial Foundation, and three times from the Adolph C. and Mary Sprague Miller Institute for Basic Research in Science. He was named California Scientist of the Year in 1981, and Berkeley Faculty Research Lecturer in 1991. He received the Button Prize of the Institute of Physics (U.K.) for Outstanding Contributions to the Science of Electromagnetic Spectrum in 1997 and the Frank Isakson Prize of the American Physical Society for Optical Effects in Solids in 2000. 